\title{
Surface Figure Metrology for CELT Primary Mirror Segments
}

G. E. Sommargren, D. W. Phillion, L. G. Seppala, S. A. Lerner

February 27, 2001 


\section{DISCLAIMER}

This document was prepared as an account of work sponsored by an agency of the United States Government. Neither the United States Government nor the University of California nor any of their employees, makes any warranty, express or implied, or assumes any legal liability or responsibility for the accuracy, completeness, or usefulness of any information, apparatus, product, or process disclosed, or represents that its use would not infringe privately owned rights. Reference herein to any specific commercial product, process, or service by trade name, trademark, manufacturer, or otherwise, does not necessarily constitute or imply its endorsement, recommendation, or favoring by the United States Government or the University of California. The views and opinions of authors expressed herein do not necessarily state or reflect those of the United States Government or the University of California, and shall not be used for advertising or product endorsement purposes.

This work was performed under the auspices of the U. S. Department of Energy by the University of California, Lawrence Livermore National Laboratory under Contract No. W7405-Eng-48.

This report has been reproduced directly from the best available copy.

Available to DOE and DOE contractors from the Office of Scientific and Technical Information

P.O. Box 62, Oak Ridge, TN 37831

Prices available from (423) 576-8401 http://apollo.osti.gov/bridge/

Available to the public from the National Technical Information Service

U.S. Department of Commerce 5285 Port Royal Rd., Springfield, VA 22161 http://www.ntis.gov/

OR

Lawrence Livermore National Laboratory Technical Information Department's Digital Library http://www.Ilnl.gov/tid/Library.html 


\section{Introduction}

The University of California and California Institute of Technology are currently studying the feasibility of building a $30-\mathrm{m}$ segmented ground based optical telescope called the California Extremely Large Telescope (CELT). The early ideas for this telescope were first described by Nelson and Mast ${ }^{1}$ and more recently refined by Nelson. ${ }^{2}$. In parallel, concepts for the fabrication of the primary segments were proposed by Mast, Nelson and Sommargren ${ }^{3}$ where high risk technologies were identified. One of these was the surface figure metrology needed for fabricating the aspheric mirror segments.

This report addresses the advanced interferometry that will be needed to achieve $15 \mathrm{~nm}$ rms accuracy for mirror segments with aspheric departures as large as $35 \mu \mathrm{m}$ peak-to-valley. For reasons of cost, size, measurement consistency and ease of operation we believe it is desirable to have a single interferometer that can be universally applied to each and every mirror segment. Such an instrument is described in this report.

\section{Background}

The primary mirror of CELT is a $30-\mathrm{m}$ diameter mosaic of hexagonal segments shown in Figure 1. Each hexagonal segment is approximately one meter across a long diameter. There are 1080 segments, accounting for the center 19 segments that are missing to accommodate the $3.6 \mathrm{~m}$ diameter secondary mirror. With 1080 segments and six-fold symmetry there are 180 different segments types, each with a unique aspheric prescription.

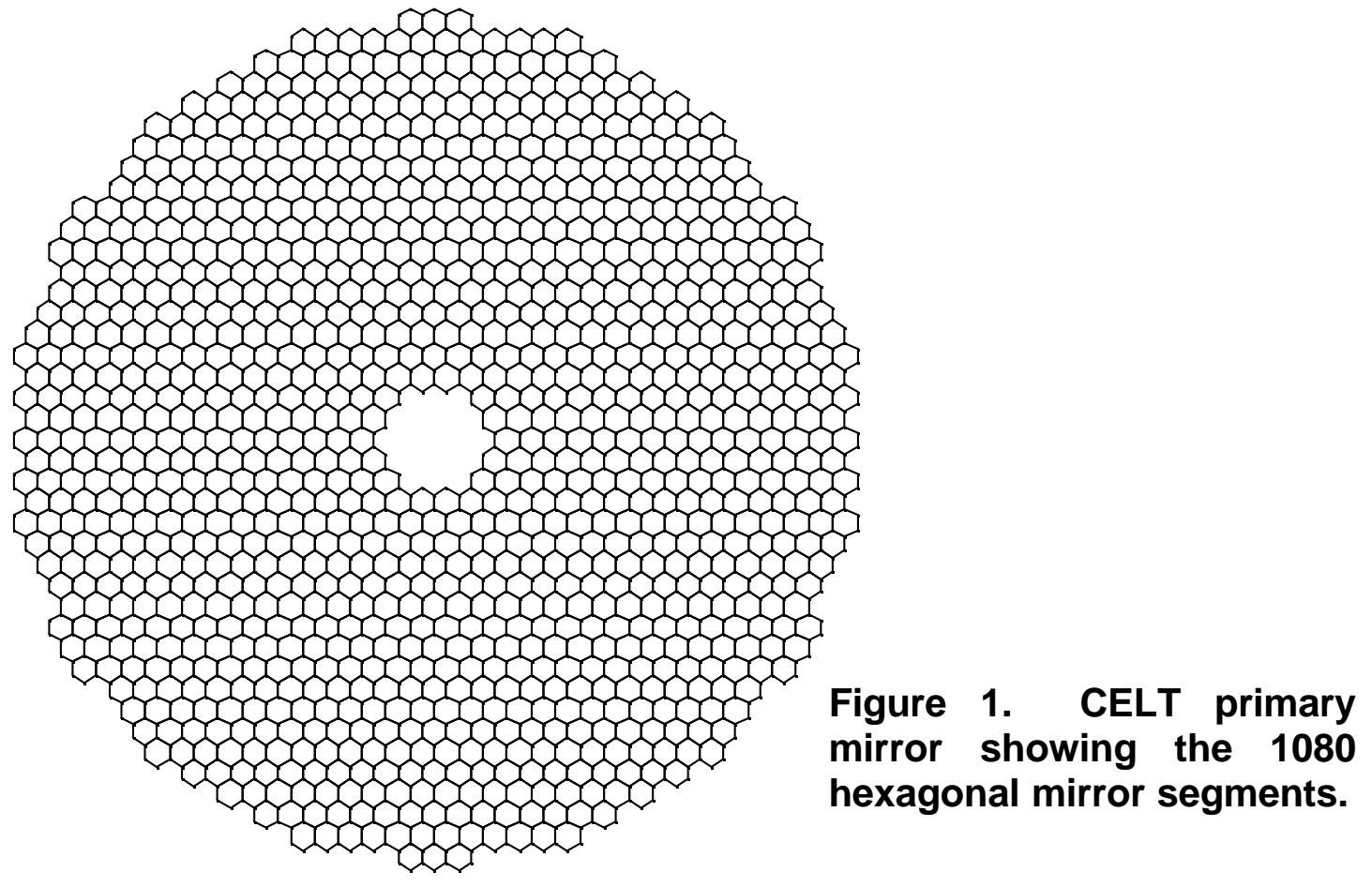


The coordinate system used to identify the individual hexagonal segments is shown in Fig. 2.

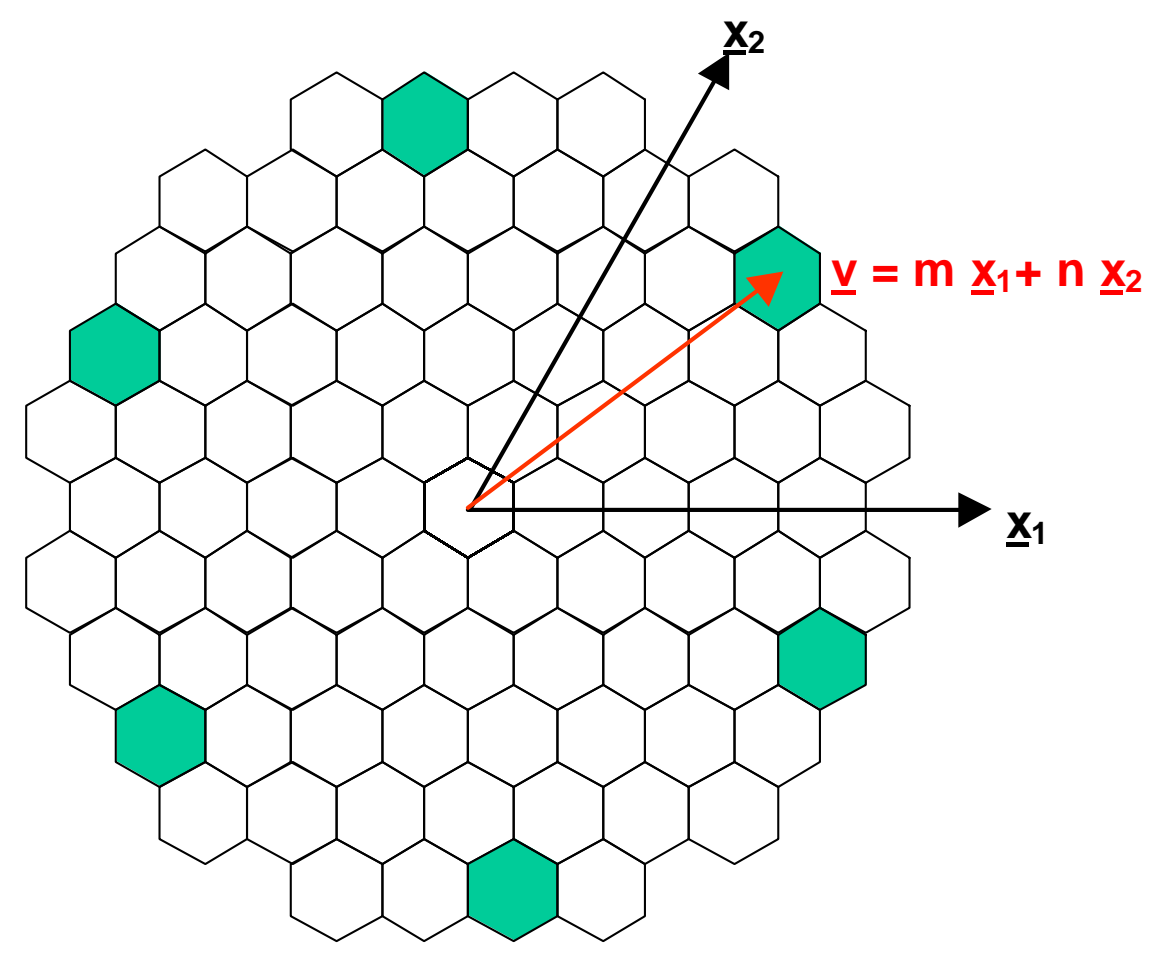

Figure 2. Coordinate system used to identify each individual segment, designated by an integer pair [m,n].

Each segment can be identified by a unique integer pair $[\mathbf{m}, \mathbf{n}]$. In general $\mathbf{m}$ and $\mathbf{n}$ can be positive or negative. However, because there is six-fold symmetry the aspheric prescription for segment $[\mathrm{m}, \mathrm{n}]$ is also the prescription for five other segments:

$$
\begin{aligned}
& \text { segment }[(m+n),-m] \\
& \text { segment }[n,-(m+n)] \\
& \text { segment }[-m,-n] \\
& \text { segment }[-(m+n), m] \\
& \text { segment }[-n,(m+n)]
\end{aligned}
$$

The six identical segments are shown shaded it the figure. Therefore, a pair of positive integers can be used to identify each of the 180 unique segments. This pair also defines the radial position of the segment center given in terms of [m,n] by,

$$
\begin{aligned}
r[m, n] & =s[\underline{v} \cdot v]^{1 / 2} \\
& =s\left(m^{2}+n^{2}+m n\right)^{1 / 2}
\end{aligned}
$$


where $\mathbf{s}$ is the width of a single hexagonal segment. This numbering system is a simple way of keeping track of a large number of segments during fabrication and final assembly.

Since each segment of the CELT primary is a section of a conic surface of revolution, the aspheric departure of each segment is dominated by the difference in the primary and secondary principal radii of curvature at the center of the segment. The primary radius of curvature $\mathbf{R}_{\mathbf{1}}$ is in the plane defined by the optical axis and the segment normal. The secondary radius of curvature $\mathbf{R}_{\mathbf{2}}$ is in a plane perpendicular to the first and containing the segment normal. The general conic surface of revolution is given by,

$$
z(r)=c r^{2} /\left(1+\left(1-(k+1) c^{2} r^{2}\right)^{1 / 2}\right),
$$

where $\mathbf{z}(\mathbf{r})$ is the surface departure at radial coordinate $\mathbf{r}, \mathbf{c}$ is the curvature at the origin $\left(R_{1}=R_{\mathbf{2}}=\mathbf{1} / \mathbf{c}\right.$ at the origin) and $k$ is the conic constant. The principal radii of curvature are given by,

$$
\begin{aligned}
R_{1} & =\left(1+z^{\prime}(r)^{2}\right)^{3 / 2} / z^{\prime \prime}(r) \\
& =(1 / c) \frac{\left(1-k c^{2} r^{2}\right)^{1 / 2}\left(1+c^{2} r^{2}\left(1-(k+1) c^{2} r^{2}\right)^{-1}\right)}{\left(1+c^{2} r^{2}(k+1)\left(1-(k+1) c^{2} r^{2}\right)^{-1}\right)}
\end{aligned}
$$

and

$$
\begin{aligned}
R_{2} & =r\left(1+z^{\prime}(r)^{2}\right)^{1 / 2} / z^{\prime}(r) \\
& =(1 / c)\left(1-k c^{2} r^{2}\right)^{1 / 2}
\end{aligned}
$$

The principal radii of curvature are plotted in Fig. 3 for $\mathbf{1 / c}=90 \mathrm{~m}$ and $\mathbf{k}=\mathbf{- 1 . 0 0 2 5}$ (preliminary value for CELT) and $\mathbf{0}=\mathbf{r}=\mathbf{1 5 m}$. The secondary radius $\mathbf{R}_{\mathbf{2}}$ is smaller and is the distance from the aspheric surface to the optical axis along the normal. The difference in radii produces astigmatism. Simulated interference patterns, generated by placing a point source at the mean center of curvature $\left(\left(\mathbf{R}_{\mathbf{1}}+\mathbf{R}_{\mathbf{2}}\right) / \mathbf{2}\right)$ of a segment, are shown in Fig. 4. The dominate aberration is astigmatism with a small amount of coma. The magnitude of astigmatism varies quadratically with $\mathbf{r}$, while coma (about an order of magnitude smaller) varies linearly with $\mathbf{r}$. The departure from a best-fit sphere varies from about $1.0 \mu \mathrm{m} p$ - $v$ for a segment near the central obscuration to nearly $35 \mu \mathrm{m} p-v$ for a segment at the outer edge. Interferometric testing will have to accommodate this large aspheric departure while maintaining an accuracy of $15 \mathrm{~nm}$ rms. 


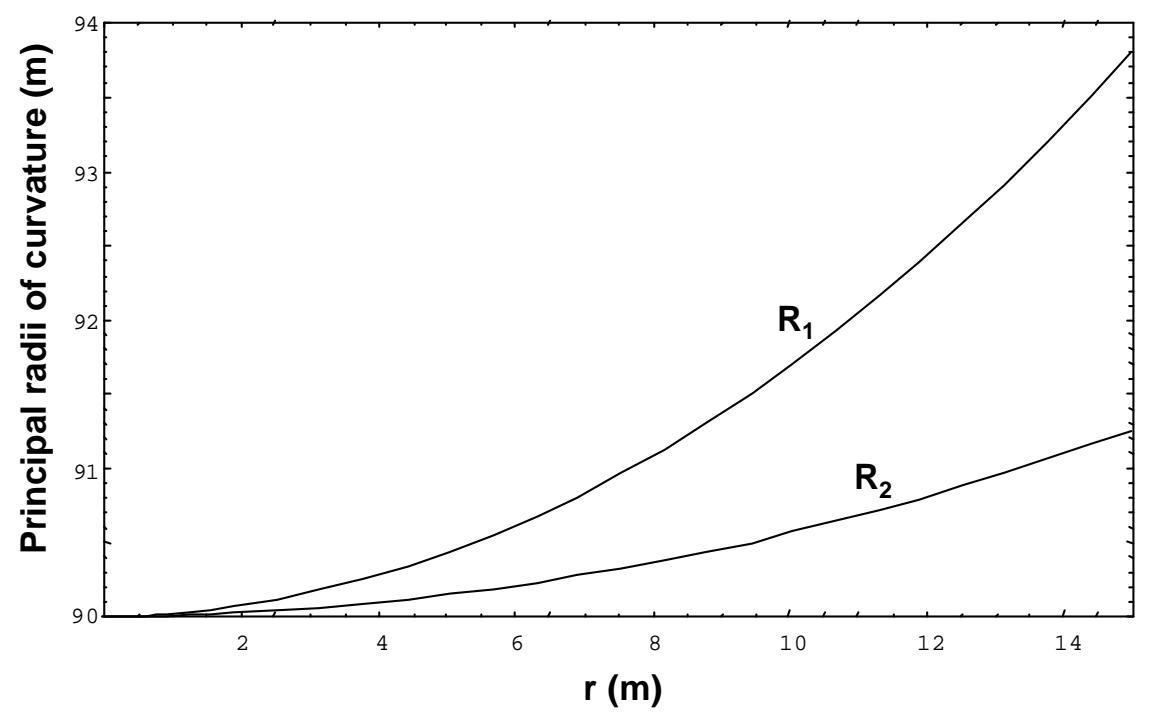

Figure 3: Principal radii of curvature of the CELT primary as a function of radius. The difference is responsible for the astigmatism in each segment.

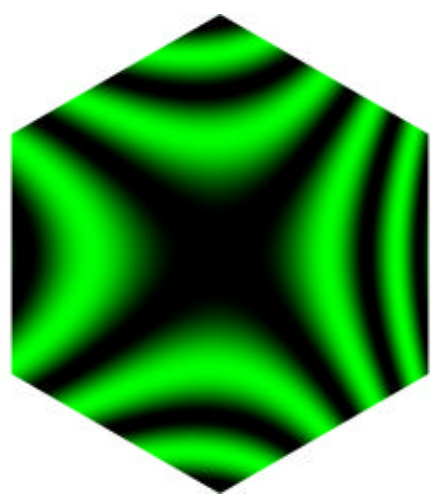

Segment $[3,0]$

$\mathrm{r}[3,0]=2.598 \mathrm{~m}$

$2.302 \mu \mathrm{m} \mathrm{p}-\mathrm{v}$

$0.414 \mu \mathrm{m}$ rms

(a)

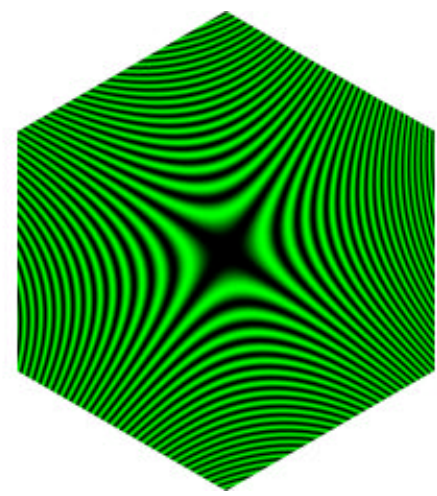

Segment $[8,2]$

$\mathrm{r}[8,2]=7.937 \mathrm{~m}$

$19.986 \mu \mathrm{m} \mathrm{p}-\mathrm{v}$

$3.691 \mu \mathrm{m} \mathrm{rms}$

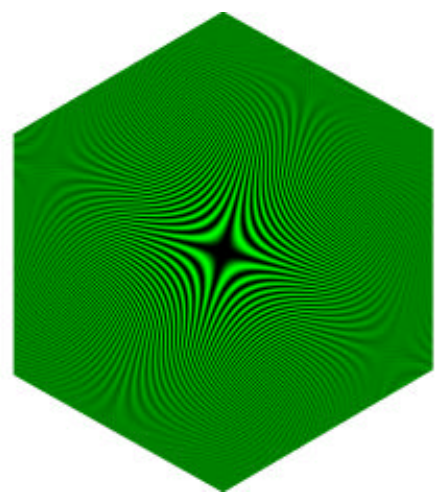

Segment $[10,10]$

$\mathrm{r}[10,10]=15.00 \mathrm{~m}$

$68.298 \mu \mathrm{m} \mathrm{p}-\mathrm{v}$

$12.870 \mu \mathrm{m} \mathrm{rms}$

(b)

(c)

Figure 4: Simulated interference patterns for (a) an inner segment; (b) a mid-radius segment; and (c) an outer segment. 


\section{Aspheric interferometry}

Aspheric figure interferometry is a special subset of general figure interferometry. Measuring the figure of a spherical surface is relatively forgiving when it comes to misalignment, coordinate mapping, magnification and calibration of the interferometer. Measuring aspheres requires an extra degree of care. Potential error sources that are overlooked or not addressed properly will result in fabrication of an incorrect asphere. The primary sources of error in aspheric interferometry are inaccurate characterization of the interferometer optics, misalignment of the interferometer components (relative to each other and to the optic under test), and errors introduced by the imaging system.

There are two coordinate systems associated with the primary mirror as shown in Fig. 5. The first is the parent coordinate system in which Eq. (2) defines the surface. In this coordinate system the z-axis is the axis of revolution and the optical axis of the primary mirror. The other is the local coordinate system of a particular segment defined by the normal at the center of the segment.

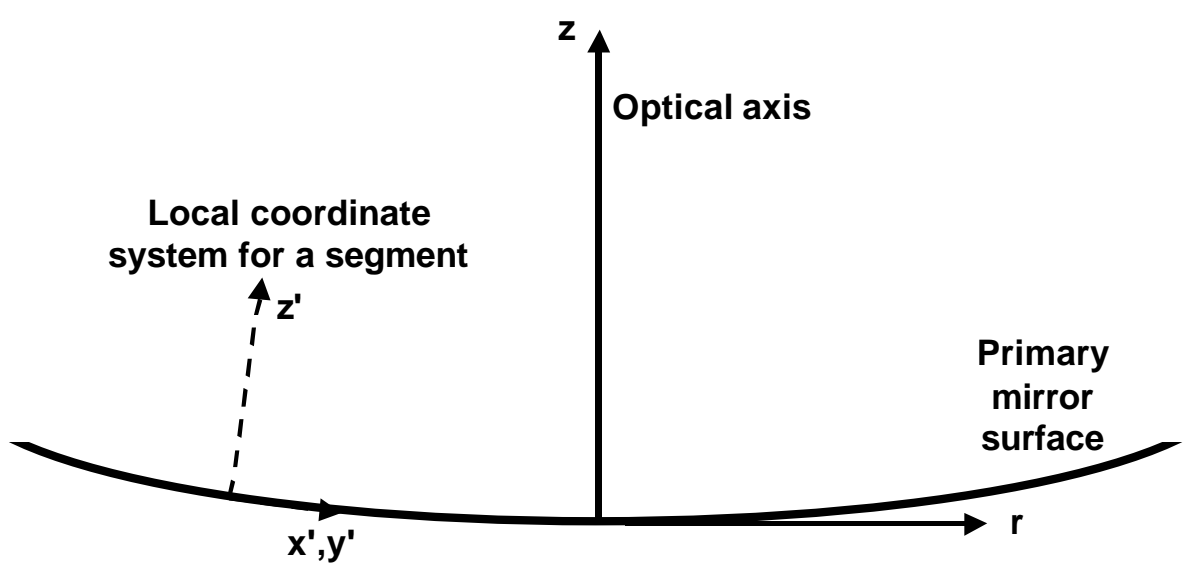

Figure 5: Coordinate systems for the primary mirror and a individual mirror segment.

Surface figure of an aspheric segment is measured in its local coordinates. This simplifies the imaging system that maps the mirror surface onto the CCD detector array. The imaging system is therefore an integral part of the interferometer and can introduce a number of errors if it is not designed properly. Design issues include:

1. Differential optical path errors due to the non-common path of the measurement and reference wavefronts for an aspheric segment;

2. Distortion of the local mirror coordinate system in the detector plane; 


\section{Magnification calibration.}

Experience has shown that differential optical path errors can be kept to about a nanometer for aspheric departures comparable to the CELT segments using a three-element imaging lens. Coordinate mapping errors due to geometric uncertainties, distortion and incorrect magnification translate directly to aspheric measurement errors. The magnitude of the error is proportional to the slope of the aspheric departure. The imaging system must therefore be designed for minimum distortion. Distortion, however, is always present at some level due to residual distortion in the lens design, and fabrication and alignment errors of the imaging lens. To remove all doubt it is prudent to measure the distortion and magnification directly. This can be done with a calibrated grid of fiducials. The calibration grid takes the form of either a mask that is placed on the surface of the mirror or a reflective deposition on the mirror. The image of this grid on the CCD provides the mapping between the mirror coordinates and the CCD pixels. This mapping is stored and used during data analysis to assign phase measurements at the CCD to the correct location on the mirror surface.

Interferometric approach: Monitoring the aspheric surface figure of each hexagonal segment during fabrication is a formidable challenge. It would be most desirable to develop one metrology that had sufficient dynamic range to accommodate all aspheric segments. This would provide continuity to the testing, minimize the effect of any residual systematic errors that may be present and keep costs to a minimum.

Over the past several years a new type of interferometry, called phase shifting diffraction interferometry ${ }^{4}$ (PSDI), has been developed that can measure off-axis aspheric mirrors with sub-nanometer accuracy. This interferometry has been used successfully to fabricate extreme ultraviolet lithographic camera mirrors with a surface figure accuracy of $0.25 \mathrm{~nm}$ rms. PSDI is based on generating wavefronts by diffraction. Diffraction is a fundamental process that permits the generation of near-perfect spherical wavefronts over a specific numerical aperture by using a circular aperture with a radius comparable to the wavelength of light $\lambda$. For example, if the aperture has a radius of $2 \lambda$ then the deviation of the diffracted wavefront from spherical is better than $\lambda / 10^{4}$ over a numerical aperture (NA) of 0.2 (20\% intensity points) in the far field of the aperture. Using this principle, two independent wavefronts can be generated - one serves as the measurement wavefront and is incident on the optic or optical system under test and the other serves as the reference wavefront. Since they are generated independently their relative amplitudes and phases can be controlled, providing contrast adjustment and phase shifting capability. This concept can be implemented in several different ways using lithographically generated apertures or single mode optical fibers.

Fig. 6 shows the PSDI configured for measuring the surface figure of a concave

off-axis aspheric mirror (optic under test). The light source is a short coherence 
length laser operating at $\lambda=532 \mathrm{~nm}$. The output beam is divided into two equal intensity beams by a polarization beamsplitter. One beam is reflected from a retroreflector mounted on a piezoelectric phase shifter and the other beam is reflected from a retroreflector mounted on a variable delay line. The two beams are recombined by the polarization beamsplitter and launched into a single mode optical fiber. The end of the fiber is imaged onto the diffraction aperture which is placed at the center of curvature of the mirror. The spherical wavefronts diffracted from the aperture have sufficient numerical aperture to illuminate both the mirror and imaging lens. The delay path-length is set equal to the round-trip distance from the fiber to the mirror. The phase-shifted wavefront reflected from the mirror is focused back onto the diffraction aperture as shown in detail in Fig. 7. It is reflected from the semi-transparent metallic coating, combining with the delayed diffracted wavefront. Since the optical path difference between these wavefronts is near zero $(<1.0 \mathrm{~mm})$ the wavefronts are temporally coherent and interfere. Extraneous wavefronts from the interferometer are temporally incoherent and do not interfere with the primary wavefronts. They do however produce a background and reduce the fringe visibility of the interfering wavefronts. A set of interference patterns is captured with a CCD camera in the image plane of the aspheric mirror as the phase is varied in steps of $\pi / 4$. The surface figure is calculated is from this data set.

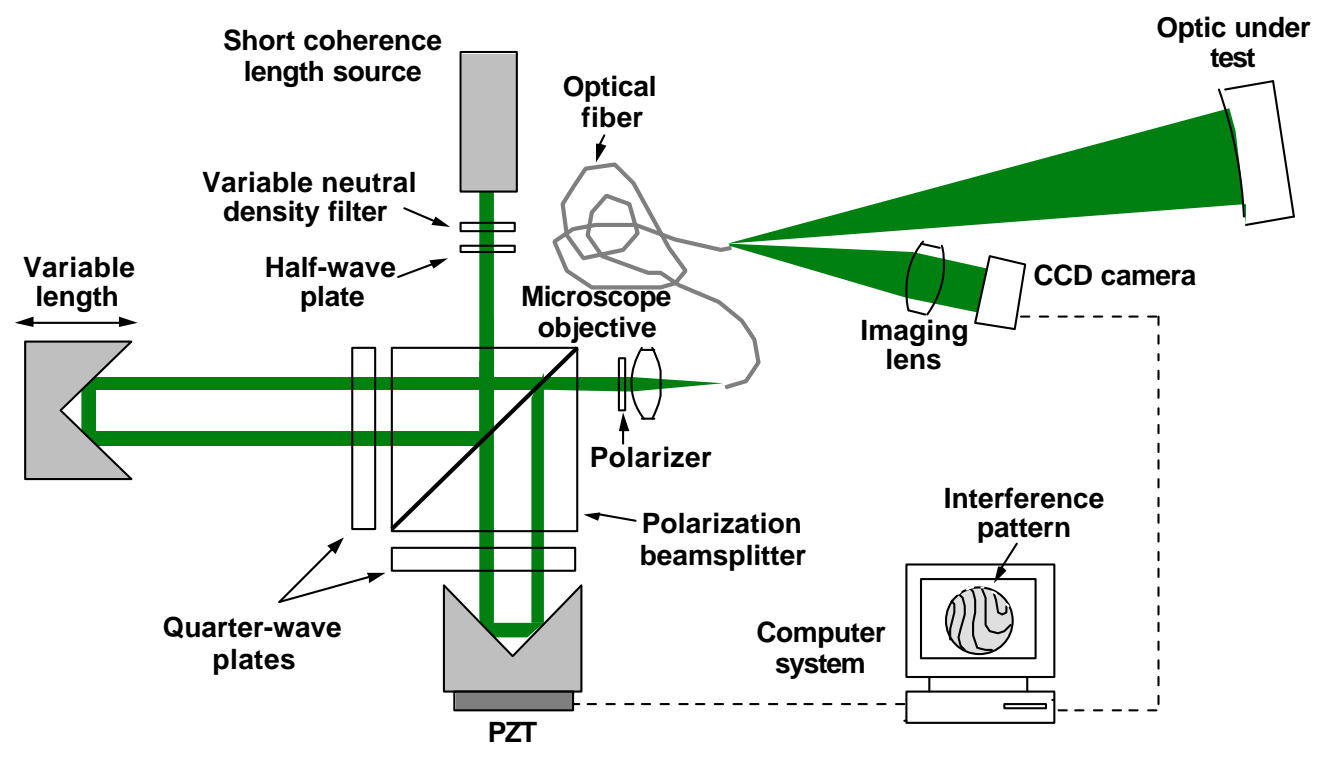

Fig. 6. PSDI configured to measure the surface figure of a concave off-axis aspheric mirror. 


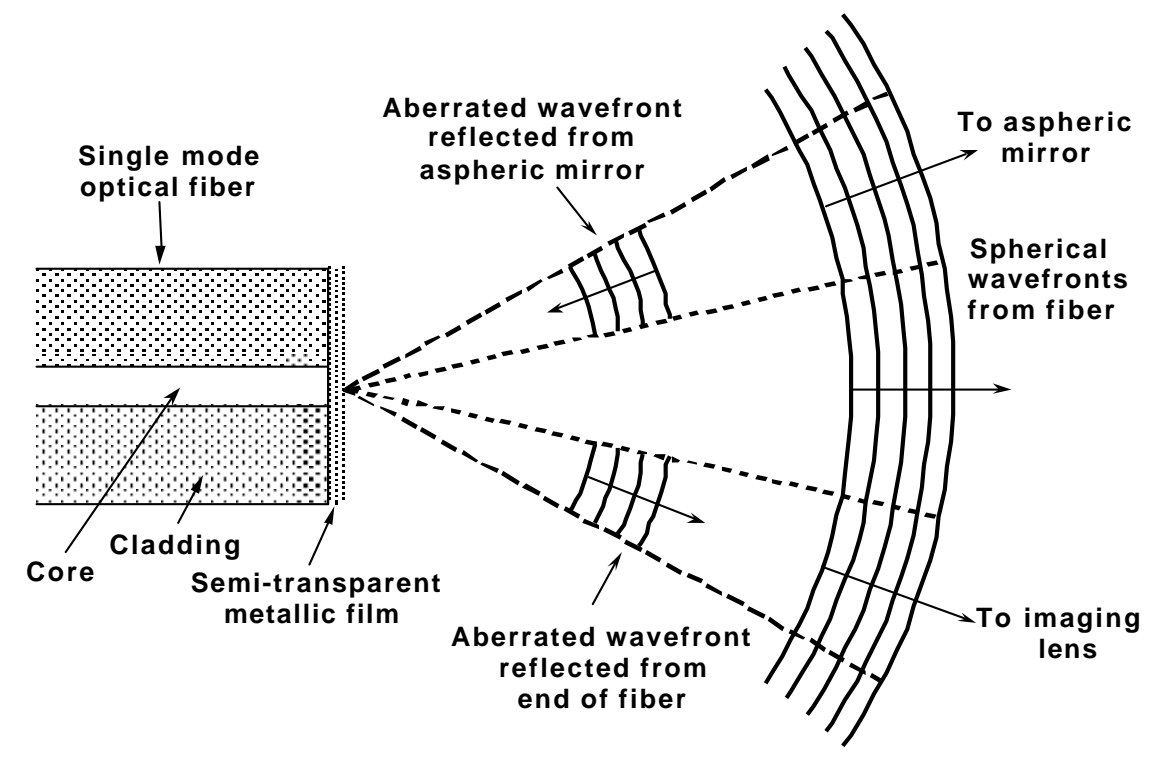

Fig. 7. Detail of the diffracted and reflected wavefronts at the end of the fiber. The principle is the same for a lithographically generated aperture.

The PSDI for measuring the CELT primary segments will require a converging lens to keep the overall length of the interferometer reasonable. The converging lens will be placed approximately $5 \mathrm{~m}$ from the diffracting aperture. This will reduce the length of the PSDI so that it can be mounted on a single optical table. The PSDI with the converging lens is shown in Fig. 8. The radius of curvature of the wavefront leaving the converging lens is approximately $90 \mathrm{~m}$. The individual primary segments are then placed in close proximity to the converging lens for testing.

Additionally, the PSDI must be able to accommodate every CELT segments even though the mean radius of curvature of the different segments varies between 90.07 and $92.52 \mathrm{~m}$. This is accomplished by accurately translating the individual segments to their correct mean radius of curvature position using a stage equipped with a distance measuring interferometer. The zero position of the stage is at the nominal $90 \mathrm{~m}$ radius of curvature as determined with a spherical reference standard. This is shown in Fig. 9 for the two extreme segments $[3,0]$ and $[10,10]$. 


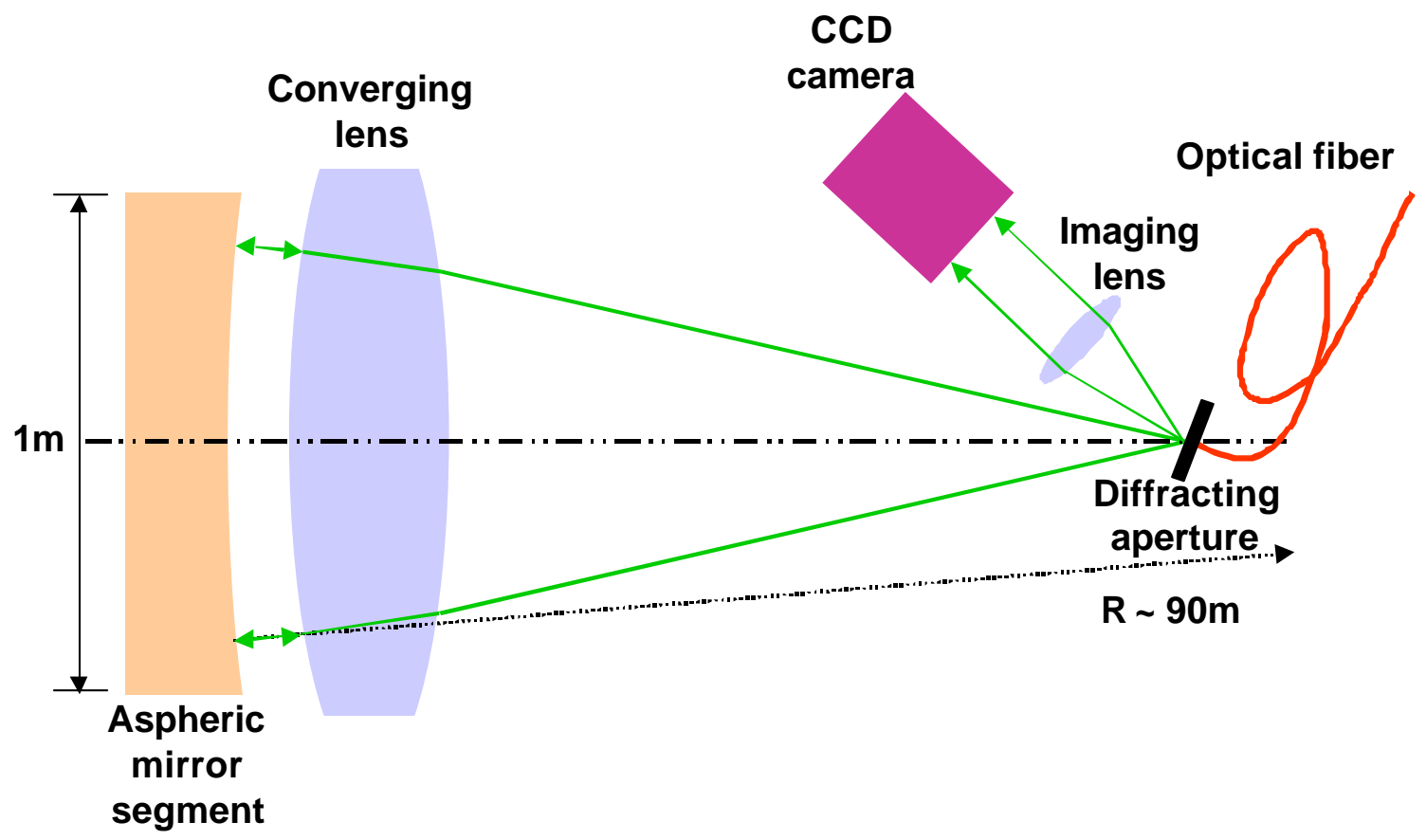

Fig. 8. PSDI configuration with converging lens to reduce the overall length of the interferometer.

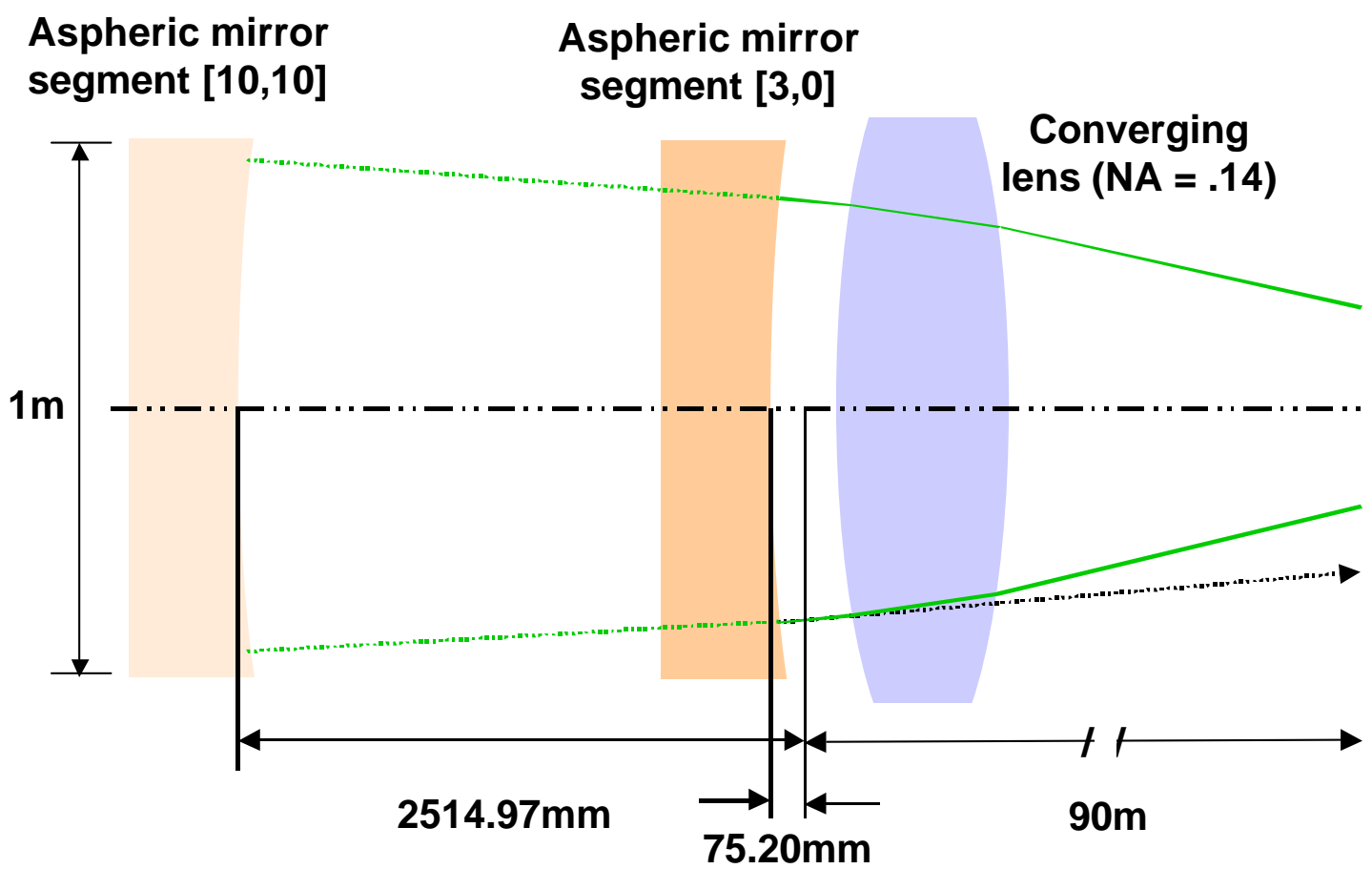

Fig. 9. PSDI configuration for measuring the two extreme CELT segments. 
Specific advantages of this method are:

a. the number of optical components is minimal, reducing the possible sources of error;

b. this configuration can address all 180 unique aspheric prescriptions in one setup. The spacing between the converging lens and mirror will require calibration to accommodate the range of the radii of curvatures for the different segments;

c. the surface figure measurement includes errors in the converging lens. However, the converging lens errors can be measured independently for compensation using a spherical surrogate mirror as described in the appendix;

d. the maximum fringe frequency is compatible with sampling the entire interferogram with a $1024 \times 1024$ pixel array.

This interferometric test is attractive in its simplicity and the ability to measure all aspheric mirror segments with a single setup. Calibration of the converging lens and measurement of the magnification and distortion are the primary error sources, but standard tests and crosschecks can be performed as described later.

\section{Design of auxiliary optical elements}

The auxiliary optical components are the converging lens and the imaging lens. These two components, as a system, must image the test mirror segment onto the CCD camera with a prescribed magnification ( $m \sim 1 / 74)$ and with minimum distortion. A numerical aperture, NA, can be defined as the maximum deviation, due to the aspheric departures, of the reflected rays from the test mirror. This NA of $0.0015(0.011$ at the CCD) is proportional to the maximum aspheric departure from all segments which are to be measured. Within this NA the wavefront errors of the imaging system over the full test diameter should be much less than the required aspheric measurement accuracy of $15 \mathrm{~nm} \mathrm{rms}$. The central or chief rays should be parallel or telecentric to minimize magnification errors due to defocus. It is important to establish accurately that the mirror is precisely imaged onto the CCD camera, because any defocus errors will lead to uncertainties in the aspheric measurement. A grid of points on a surrogate mirror can be used to accurately determine lateral distortion and exact focus, provided that the wavefront errors of this imaging system are less than $\lambda / 4$ over a numeric aperture of three times NA. In this way, focus can be set to better than $10 \%$ of the Raleigh depth of focus for the aspheric measurement.

Converging lens - design considerations and configurations: An auxiliary converging lens, shown schematically in Figs. 8 and 9 , is used to reduce the length of the PSDI from about 90 meters to approximately 7 meters. A tradeoff is 
necessary in choosing the focal length of the converging lens. A long focal length converging lens is easier to correct for aberrations. On the other hand, a short focal length converging lens reduces the distance between the fiber and the mirror, reducing the cost associated with the tower, air turbulence and mechanical stability. However, it also requires a very small diffracting aperture. A distance of 5 meters between the diffracting aperture and the converging lens has been chosen, for an overall length for the PSDI of about 7 to 8 meters.

The converging lens will also be measured in double pass along with the test mirror segment. Therefore, the transmitted wavefront error for the spherical diffracted beam should be sufficiently small to avoid errors during the subtraction process.

Several options for the $1.0 \mathrm{~m}$ diameter converging lens have been considered. All lenses are assumed to be BK7, although fused silica is also an option. All the converging lens options can be designed to meet the above wavefront and imaging conditions.

1. Singlet using aspheric surface: The simplest converging is a plano-convex aspheric singlet. The aspheric surface is described using a radius of curvature, conic constant, and $6^{\text {th }}$ order aspheric term. These terms define the focal length of the lens, the contribution to $4^{\text {th }}$ order spherical aberration, and the contribution to $6^{\text {th }}$ order spherical aberration respectively. As field curvature and astigmatism are the dominant aberrations adding more aspheric terms will not improve the design. The convex surface has a maximum departure of $74 \mu \mathrm{m}$ from a best-fit sphere. A singlet made of silica will be aplanatic if the ratio of the radius of curvature of the surface facing the mirror to the surface facing the fiber is about 1:7. The advantage is marginally looser alignment tolerances. The maximum aspheric departure of the aplanatic design is $68 \mu \mathrm{m}$.

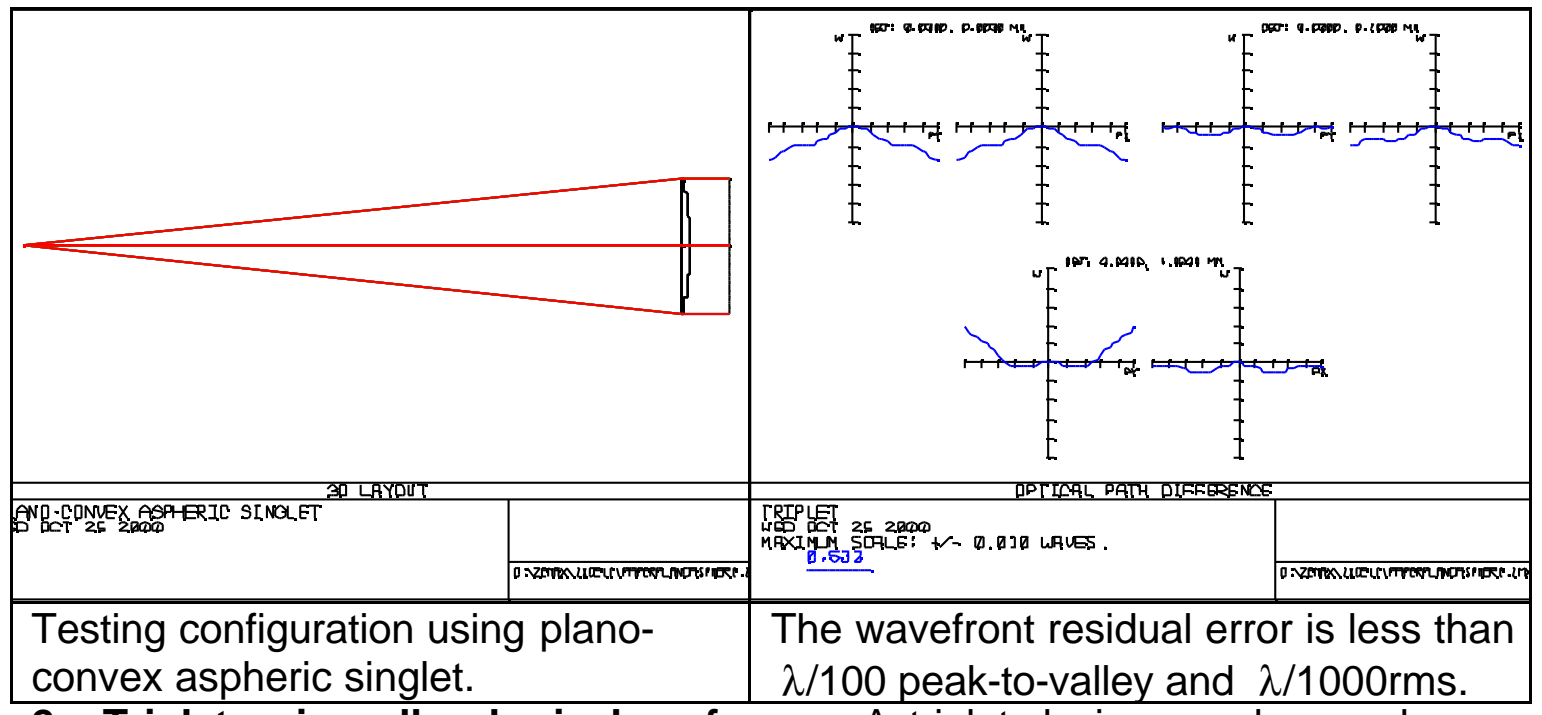

2. Triplet using all spherical surfaces: $A$ triplet design can be used as an aplanatic converging lens. The primary advantage of such a configuration is that 
is uses all spherical surfaces. Testing and fabrication of the individual lenses may be less costly. Each lens of the triplet design is approximately $70 \mathrm{~mm}$ thick. Light therefore travels through more than $400 \mathrm{~mm}$ of glass in the double pass configuration. Assuming an index homogeneity of $0.5 \mathrm{ppm}$, the surface contribution to the peak to valley wavefront error will be about $\lambda / 2$. The nominal wavefront error is negligible.

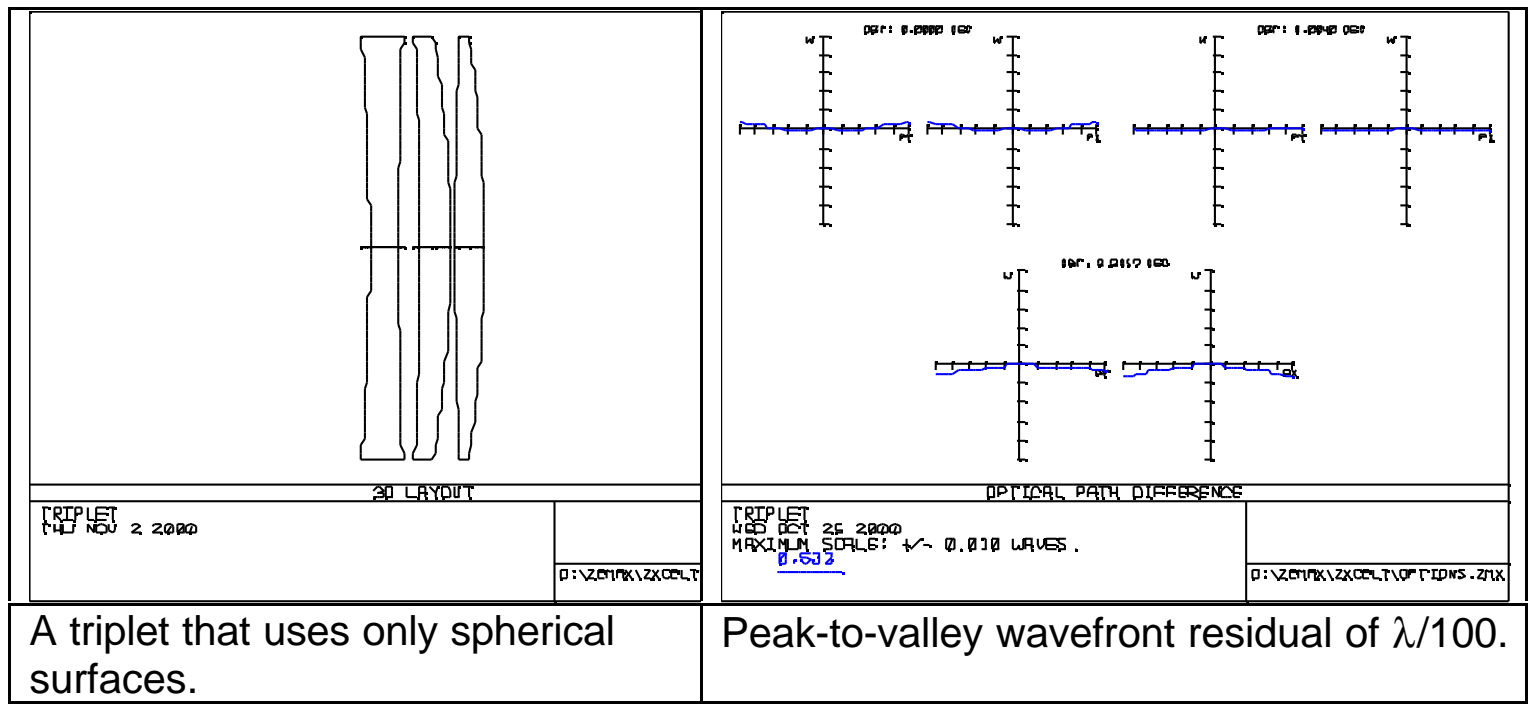

3. Condenser/compensator: A configuration that uses a condenser lens in combination with an aspheric singlet placed close to the fiber combines some advantages of the aspheric singlet design and the spherical triplet design. In this configuration the compensator has a clear aperture of $370 \mathrm{~mm}$ and a maximum asphericity of $48 \mu \mathrm{m}$. The thickness of the glass used is about $200 \mathrm{~mm}$, which is half of that used in the triplet design.

\begin{tabular}{|c|c|c|}
\hline \multicolumn{2}{|c|}{$\sim 1$} & (a) \\
\hline 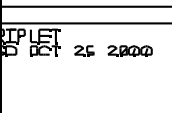 & & 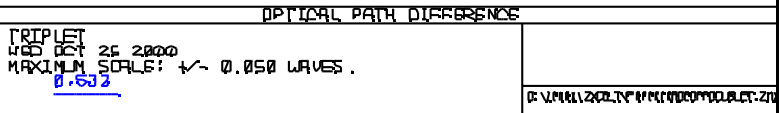 \\
\hline $\begin{array}{l}\text { Spherica } \\
\text { aspheric }\end{array}$ & $\begin{array}{l}\text { with an } \\
\text { ating lens. }\end{array}$ & $\begin{array}{l}\text { Peak-to-valley wavefront residual of } \\
\lambda / 40\end{array}$ \\
\hline
\end{tabular}


A spherical doublet can be used as the compensator instead of the aspheric singlet. A representative design shown below uses a doublet corrector with a clear aperture of $300 \mathrm{~mm}$ and has a peak-to-valley wavefront residual of $\lambda / 100$. The meniscus lens will be challenging to fabricate to the necessary tolerances. Allowing for a doublet with a larger clear aperture enables a loosening of the tolerances on the doublet. The design uses about $300 \mathrm{~mm}$ thickness of glass.

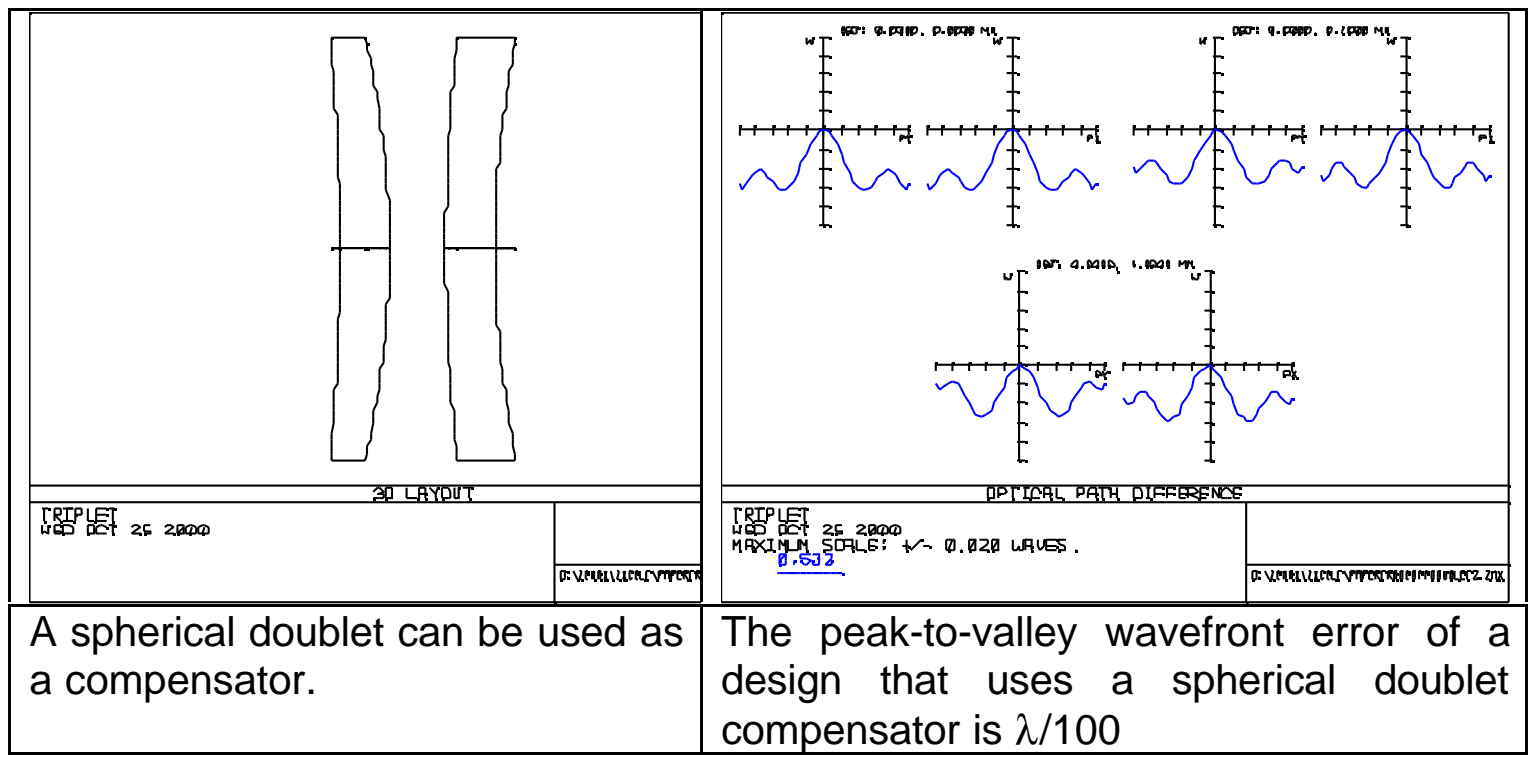

Comparison: Representative design strategies have been studied and the results summarized in the table below. As the requirements become better defined a choice between the various configurations can be made. Additional tradeoffs between performance, tolerances and cost can also be made.

\begin{tabular}{|l|c|c|c|}
\hline & $\begin{array}{l}\text { Aspheric } \\
\text { singlet }\end{array}$ & Triplet & $\begin{array}{l}\text { Condenser/ } \\
\text { Doubet } \\
\text { Compensator }\end{array}$ \\
\hline $\begin{array}{l}\text { Nominal peak-to- } \\
\text { valley OPD residual } \\
\text { error }\end{array}$ & $<\lambda / 100$ & $<\lambda / 100$ & $<\lambda / 100$ \\
\hline Number of surfaces & 2 & 6 & 6 \\
\hline $\begin{array}{l}\text { Thickness of glass } \\
\text { that light travels } \\
\text { through (double } \\
\text { pass configuration) }\end{array}$ & $140 \mathrm{~mm}$ & $420 \mathrm{~mm}$ & $280 \mathrm{~mm}$ \\
\hline $\begin{array}{l}\text { Maximum } \\
\text { asphericity }\end{array}$ & $74 \mu \mathrm{m}$ & $\mathrm{N} / \mathrm{A}$ & $\mathrm{N} / \mathrm{A}$ \\
\hline Weight & $75 \mathrm{~kg}$ & $306 \mathrm{~kg}$ & $87 \mathrm{~kg}$ \\
\hline
\end{tabular}


Imaging lens - design configurations: Besides meeting the imaging requirements, the imaging lens should produce a perfect plane wavefront from the input spherical reference wavefront. Slight defocus errors then do not introduce mapping errors or contribute to measurement errors. A three-element lens can meet the necessary requirements. The diameter of the imaging lens is less than $25 \mathrm{~mm}$. A four-element lens can be designed with features that minimize the fabrication and assembly difficulties, with negligible cost impacts, and is shown in Fig. 10.

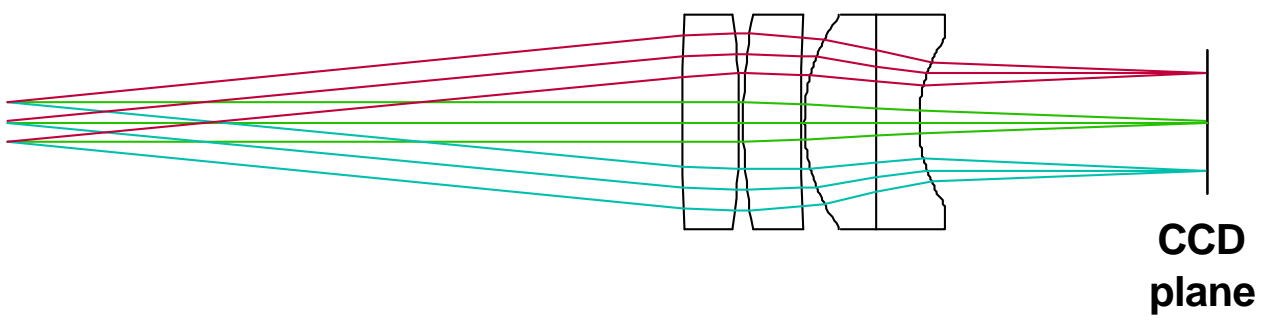

Fig. 10. A four-element imaging lens is the best choice for fabrication, assembly and cost.

\section{Prerequisites for meeting accuracy requirements}

A spherical surrogate mirror of nominally 91 meters radius will be used to measure the non-shear interferometer errors so that they can be corrected for in the analysis software. It will also be used to position each segment so that its mean radius of curvature can be measured.

We believe that we can measure absolutely the average radius of curvature of a segment to at least $1 \mathrm{~mm}$ or $1 \times 10^{-5}$ fractional accuracy and possibly to $0.5 \mathrm{~mm}$ or $5 \times 10^{-6}$ fractional accuracy if a high enough quality spherical surrogate mirror can be obtained. What limits us is how accurately we can measure the radius of curvature of the spherical surrogate mirror. If it were possible to figure the 
surrogate mirror to $\lambda / 50 \mathrm{rms}$ in reflection, we could find its null position to an accuracy of at least $0.5 \mathrm{~mm}$.

We will measure the radius of curvature of the spherical surrogate mirror by moving it precisely one meter along the optical axis of the PSDI from its "best null" position and observing the resulting focus fringes. This will give maximum $x$ and $y$ fringe density components of 0.23 fringes per pixel at the edges of the $1024 \times 1024$ CCD. There will be a distortion calibration grid on the spherical surrogate mirror (discussed later) which will be used to accurately determine the magnification. There will be 58 focus fringes. Measuring the phase to an accuracy of $1 / 2000$ wave gives us a fractional accuracy of $1 \times 10^{-5}$ in the radius measurement. This can be improved by a factor of two if we go plus and minus precisely one meter. The magnification must be measured to the same accuracy. It may be noticed that the 1/2000 wave is considerably better than the figure of the spherical surrogate mirror. We will take the difference of the two interferograms after accounting for the change in magnification so that the figure of the spherical surrogate mirror subtracts out. Thus we are justified in making measurements that are much more accurate than the figure of the spherical surrogate mirror.

We will be able to do much better as far as relative average radius errors between the CELT mirror segments are concerned. We will arbitrarily choose some "best null" interferogram for the spherical surrogate mirror as corresponding to the reference position. If the mean radius of a segment is to be $R_{\text {segment }}$ and the spherical surrogate mirror radius is $R_{\text {surrogate, the mirror segment }}$ will be moved $R_{\text {segment }}-R_{\text {surrogate }}$ from the spherical surrogate mirror "best null" position. This can be done to an accuracy of a few microns by indicating off the center of the optic. So that we do not depend upon a single spherical surrogate mirror, other spherical surrogate mirrors can be referenced to the arbitrarily chosen "primary standard" spherical surrogate mirror. A one millimeter change in the focal length of the nearly paraboloid multi-segment CELT mirror will be unimportant.

What would be the most damaging would be random errors in the mean radii of curvature for the various CELT mirror segments. This would create scatter in a circle of approximately thirty times the Airy disk diameter. Even though the effect on the telescope resolution would be minuscule, the scattering would compromise detecting extra-solar planets. The procedure just described will avoid this.

There will be a distortion calibration grid (DCG) evaporated onto the spherical surrogate mirror that will be used to measure the distortion of the optical system. This is an array of evaporated aluminum crosshair fiducials whose centers can be located on the CCD to an accuracy of at least 0.02 pixels. The fiducial array will be over-coated with an array of anti-reflection coated circles. The fiducials will be brightly reflecting against a very dark background. Very high intensity 
contrast is needed because the measurement will be done using phase-shifting interferometry that essentially measures the electric field. There will be adequate area where the fiducials aren't present to find the "best null" position. We have extensive experience with measuring the distortion this way for making the EUVL projection lithography optics. The physical dimensions of each evaporated aluminum crosshair fiducial will be such that the image of each of its fiducial arms on the CCD will be 1.5 pixels wide and 50 pixels long.

We will be able to locate the center of each fiducial to about 0.02 pixel accuracy. Possibly as great as 0.01 pixel accuracy will be achieved by averaging on the corners of a $1 / 2$ by $1 / 2$ pixel grid. This will give $1 \times 10^{-5}$ fractional accuracy for the magnification from the spherical surrogate mirror for a 1024x1024 CCD. When the 2048x2048 CCD camera becomes available, we will be able to achieve a fractional accuracy of $5 \times 10^{-6}$ in measuring the magnification to the CCD from the spherical surrogate mirror. We have extensive experience both in knowing what's needed for the fiducials and in knowing how accurately the centers may be located. Perhaps the best experimental demonstration that 0.02 pixel accuracy may be obtained was achieved in the PSDI interferometers by rotating the imaging lens while imaging either a DCG or a fiducial mask. What we were trying to measure was the lens precession. For each fiducial, a trajectory was traced out. Typically the trajectories were somewhat irregular circles a few pixels across. These trajectories could be overlaid by superimposing their centroids. The points from the various fiducials matched each other to about 0.02 pixels rms.

Determining the distortion is the most critical for the outermost segments. For an outermost segment at $r=15 \mathrm{~m}$, a 0.08 pixel error for a $1024 \times 1024$ CCD occurring where the interferogram fringe density is highest and in the direction of the phase gradient will cause a $10 \mathrm{~nm}$ error in the surface height measurement. A 0.08 pixel peak error in knowing positions will cause an rms surface height error twelve times smaller than this. Where the fringe density is small near the center of the interferogram, an error in knowing the position has little effect. Also a positional error transverse to the phase gradient has no effect. Nevertheless our goal is to keep the peak surface height error due to an error in measuring the distortion less than $10 \mathrm{~nm}$. We believe this possible based upon our previous experience.

We also plan to use a magnification calibration mask (MCM). It will kinematically mount closely onto the front of the segments and will be used both to verify the magnification of the optical system and to obtain the absolute positioning. The MCM will have four fiducials located near the $+/-x$ and $y$ limits. Its fabrication cost should be negligible compared to that of the distortion calibration grid. The distortion mapping has to be re-measured each time the $z$ position changes but does not have to be re-measured while one is working on a set of identical mirror segments. A new measurement with the MCM will be made each time an optic is to be re-measured after a polishing step. All the MCM does that the DCG does 
not is to give us the absolute positioning. Other than that it is merely a convenience to verify that nothing has changed.

\section{Measuring the interferometer errors}

There are two kinds of interferometer errors, which we call shear and non-shear errors. The non-shear errors will be measured and corrected for. These are the interferometer errors that would be measured if we replaced a CELT mirror with a perfect spherical mirror having its best fit radius. The shear errors arise because the actual ray paths taken by the reflected rays from a CELT mirror differ from the reflected ray paths for the perfect spherical mirror.

We must differentiate between two kinds of shear errors. There are the shear errors which are intrinsic to the optical system and which can be predicted by CODE V. The optical system is designed so as to make these errors very small. Also, since they are known, they can be corrected for. Then there are the shear errors due to random height and slope errors in the surfaces of the lenses. If the measurement and reference rays traveled common paths, then surface height errors of the imaging lens would not matter at all and the interferometer errors caused by the converger lens could be exactly measured using the spherical mirror. We can correct for everything except for the shear errors caused by a reflected ray from a CELT mirror experiencing slightly different surface height and slope errors than the corresponding reflected ray from the spherical mirror which can be put in the interferometer in place of the CELT mirror.

Given a perfect spherical mirror, the non-shear interferometer errors are of course directly and simply measurable. The experimental problem, however, is to measure the non-shear interferometer errors given an imperfect spherical mirror. The azimuthally varying interferometer errors are simply measured as follows: If we average the measurements for the interferometer error for $\mathrm{N}$ equally spaced rotational positions of the imperfect spherical mirror assuming the spherical mirror to be perfect, we will obtain a measurement for the interferometer error that is correct except for those Zernike terms involving $\cos (m \phi)$ or $\sin (m \phi)$ for which $m$ is an integer multiple of $N$. This occurs because the geometric series:

$$
\sum_{n=0}^{N-1} \exp \left[i m\left(\phi+\frac{2 \pi n}{N}\right)\right]
$$

sums to zero whenever $\mathrm{m}$ is not an integer multiple of $\mathrm{N}$. The problem now is to measure the purely radial interferometer errors for which $m=0$.

By making a set of measurements at discrete rotational positions of the spherical surrogate mirror we can determine the non-radial Zernike coefficients for the errors in both the spherical surrogate mirror and the PSDI over a circle A. A second measurement set can be made by rotating the mirror about an off-axis 
point to get the non-radial Zernike coefficients over a circle B. Given the nonradial Zernike coefficients (except for tilts) for the spherical surrogate mirror in a circle $\mathbf{A}$ and also in a circle $\mathbf{B}$ which is off-center to and contained in circle $\mathbf{A}$, the radial Zernike coefficients except for piston and focus may be exactly reconstructed. This is described in Appendix A. Thus we measure the radial Zernike coefficients for both the interferometer error and the imperfect spherical mirror figure.

This procedure in Appendix $A$ requires that we be able to transform Zernike coefficients for one circle to Zernike coefficients for another circle with a different center and different normalizing pupil radius. Mathematical methods for doing this are described in appendices B, C, and D. Reading the first part of appendix $B$ is required for understanding appendices $C$ and $D$. Appendix $B$ introduces the notation and that part of the formalism of orthogonal polynomial function theory that we use. Translating and scaling can be done either by staying entirely in the space of Zernike functions (appendices B and C) or by transforming to multinomial function space and doing the translating and scaling there and then transforming back to Zernike function space. In fact, we have implemented both methods and of course the same answer is obtained either way.

\section{Summary}

This report addresses the challenge of measuring the surface figure of the 180 unique aspheric primary mirror segments for CELT. The proposed interferometric technique is similar to the approach that was taken to measure aspheric mirrors for extreme ultraviolet lithography projection cameras where sub-nanometer accuracy was achieved. To keep the measurement system on the scale of typical optics facilities, a converging lens will be used. Several design options were presented for this lens as well as a design for an imaging lens that is necessary to map the mirror segment surface onto the CCD. Methods to measure the radii of curvature and calibrate the interferometer were also discussed. We feel this technique has the accuracy and versatility to measure each and every aspheric segment with one just one instrument - a major advantage for consistency, cost and ease of operation.

\section{References}

1. Nelson, J.E. and Mast, T.S., "Giant optical devices," Proceedings of the Backaskog Workshop of Extremely Large Telescopes, Anderson, ed, pp. 111, June 1999, Lund University and ESO.

2. Nelson, J.E., "Design concepts for the California Extremely Large Telescope (CELT)," Proceedings of the SPIE, 4004, pp. 282-289, 2000. 
3. Mast, T.S., Nelson, J.E., and Sommargren, G.E., "Primary mirror segment fabrication for CELT," Proceedings of the SPIE, 4003, pp. 43-58, 2000.

4. Sommargren, G.E., "Phase shifting diffraction interferometry for measuring extreme ultraviolet optics," OSA Trends in Optics and Photonics Vol. 4, Extreme Ultraviolet Lithography, Kubiak and Kania, eds. (Optical Society of America, Washington, DC 1996), pp. 108-112. 
APPENDIX A: Determining the radial Zernike coefficients given the nonradial Zernike coefficients within a circle $A$ and also within a circle $B$ offcenter to and contained in the circle $A$

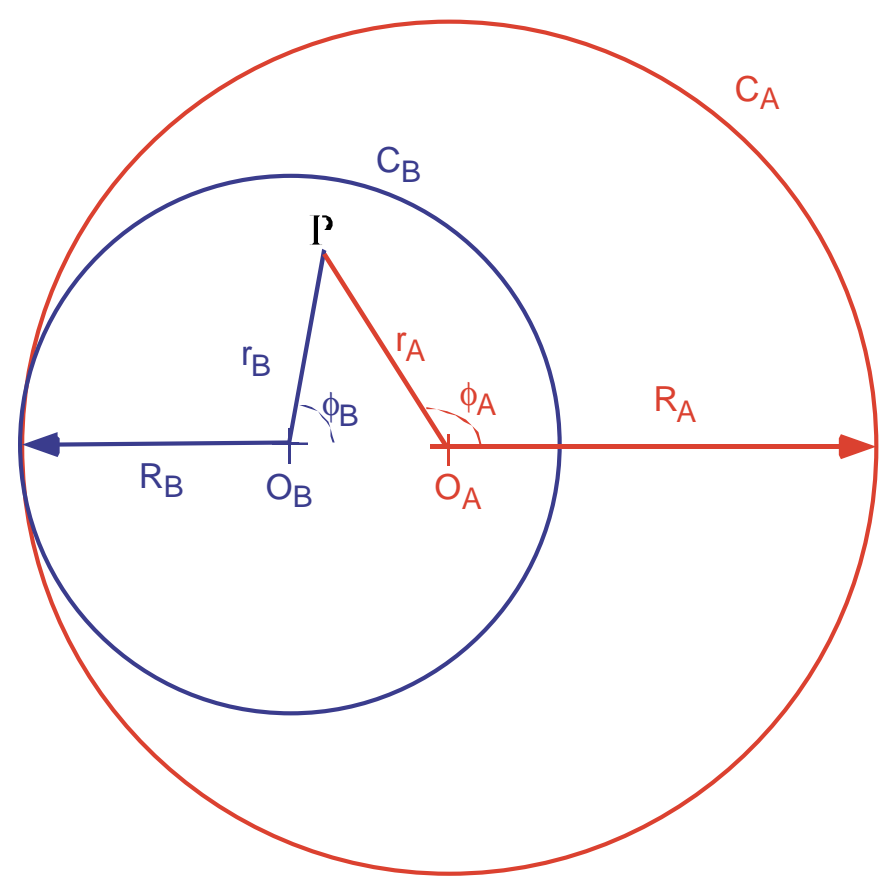

We are given the non-radial Zernike coefficients for circle $\mathbf{A}$ about its origin and with its pupil radius and for circle $\mathbf{B}$ about its origin and for its pupil radius. If the unknown radial Zernike terms are included, the two Zernike expansions must agree in their overlap region, which is all of circle $\mathbf{B}$. We thus have:

$\sum_{n} \sum_{m \neq 0} c_{m m}^{(A)} Z_{n, m}\left(\frac{r_{A}}{R_{A}}, \phi_{A}\right)+\sum_{n=0} c_{n 0}^{(A)} Z_{n, 0}\left(\frac{r_{A}}{R_{A}}, \phi_{A}\right)=\sum_{n} \sum_{m \neq 0} c_{m m}^{(B)} Z_{n, m}\left(\frac{r_{B}}{R_{B}}, \phi_{B}\right)+\sum_{n} c_{n 0}^{(B)} Z_{n, 0}\left(\frac{r_{B}}{R_{B}}, \phi_{B}\right)$

We may rewrite this as

$\sum_{n} c_{n 0}^{(A)} Z_{n, 0}\left(\frac{r_{A}}{R_{A}}, \phi_{A}\right)-\sum_{n} c_{n 0}^{(B)} Z_{n, 0}\left(\frac{r_{B}}{R_{B}}, \phi_{B}\right)=\sum_{n} \sum_{m \neq 0} c_{n m}^{(B)} Z_{n, m}\left(\frac{r_{B}}{R_{B}}, \phi_{B}\right)-\sum_{n} \sum_{m \neq 0} c_{n m}^{(A)} Z_{n, m}\left(\frac{r_{A}}{R_{A}}, \phi_{A}\right)$

(A.2)

where the unknown terms are now on the left and the known terms are now on the right. We might think to use least squares to solve for the unknown coefficients but there is one complication that must be addressed first. There may be piston, tilts and focus between the $\mathbf{A}$ and $\mathbf{B}$ measurements. Thus we really wish to do least squares with the following expansion: 


$$
\begin{aligned}
& \sum_{n \geq 2} c_{n 0}^{(A)} Z_{n, 0}\left(\frac{r_{A}}{R_{A}}, \phi_{A}\right)-\sum_{n \geq 2} c_{n 0}^{(B)} Z_{n, 0}\left(\frac{r_{B}}{R_{B}}, \phi_{B}\right)+f_{1}+f_{2} x_{A}+f_{2} y_{A}+f_{3}\left(2 r_{A}^{2}-1\right)= \\
& \sum_{n \geq 2} \sum_{m \neq 0} c_{n m}^{(B)} Z_{n, m}\left(\frac{r_{B}}{R_{B}}, \phi_{B}\right)-\sum \sum_{n \geq 2} c_{m \neq 0}^{(A)} Z_{n, m}\left(\frac{r_{A}}{R_{A}}, \phi_{A}\right)
\end{aligned}
$$

Note that only the Zernike terms for $n>=2$ have been kept. The least squares functions are the piston, the $x$ tilt, the $y$ tilt, the focus and the coefficients of the radial Zernikes for $n>=2$ for both circle $\mathbf{A}$ and circle $\mathbf{B}$. The least squares functions are on the left hand side of the equation.

This has been tested by assuming a Zernike expansion in circle $\mathbf{A}$, then finding the Zernike expansion in circle $\mathbf{B}$ using translation and scaling transformations, then by setting the coefficients in both expansions for the radial Zernikes and the $x$ and $y$ tilts to zero, and then by doing least squares fitting in circle $\mathbf{B}$. (Piston and focus are radial Zernikes). The procedure is exact when there is no noise. Since the least squares fitting gives the radial Zernike coefficients for both circles $\mathbf{A}$ and $\mathbf{B}$, the optic does not have to be made oversize in order to determine the figure within the clear aperture.

Let $\mathrm{f}_{\mathrm{i}}(\mathrm{x})$ be a set of functions for which least squares fitting of the equation

$$
\sum_{i} c_{i} f_{i}\left(x_{k}\right)=g\left(x_{k}\right) \text { (A.4) }
$$

is to be done. Here $x_{k}$ is a set of points. For our case $x_{k}$ will be a set of $(x, y)$ points on a square grid filling circle $\mathbf{B}$. The denser the grid the more accurate the result. However, I find that a $100 \times 100$ square grid masked to circle B is more than fine enough. This can be cast as the matrix equation

$$
\sum_{j} A_{i j} c_{j}=B_{i}
$$

where

$$
A_{i j}=\sum_{k} f_{i}\left(x_{k}\right) f_{j}\left(x_{k}\right)(A
$$

and

$$
B_{i}=\sum_{k} f_{i}\left(x_{k}\right) g\left(x_{k}\right)
$$

From this we see that the solution vector $\mathrm{c}_{i}$ is linear in the nonradial Zernike coefficients for $n>=2$ for circles $\mathbf{A}$ and $\mathbf{B}$. The $c_{i}$ are the radial Zernike coefficients for $n>=2$ for circles $\mathbf{A}$ and $\mathbf{B}$ and the piston, tilts, and focus.

What this means is that for each nonradial Zernike coefficient for either circle $\mathbf{A}$ or circle $\mathbf{B}$, we can construct a $\mathbf{B}$ vector which represents only that part of $\mathrm{g}(\mathrm{x})$ due to a unit value of that nonradial coefficient in Eq. (A.7). Denote this B vector by, for example,

$$
B_{i}^{(A, n, m)}
$$

where $(A, n, m)$ denotes the nonradial component with shell number $n$ and azimuthal number $\mathrm{m}$ for circle $\mathbf{A}$. What we are really interested in is the vectors 


$$
A^{-1} B_{i}^{(A, n, m)}
$$

for circle $\mathbf{A}$ and in the vectors

$$
A^{-1} B_{i}^{(B, n, m)}
$$

for circle B. They tell us the sensitivities of the solution vector to errors in the various nonradial Zernike coefficients for $n>=2$ for circles $\mathbf{A}$ and $\mathbf{B}$. I have looked at these vectors and verified that this technique for reconstructing the radial Zernike coefficients for $n>=2$ from the nonradial Zernike coefficients for $n>=2$ in circles $\mathbf{A}$ and $\mathbf{B}$ is numerically sound.

We next present a numerical example to demonstrate the numerical wellbehavedness of this method. Suppose we have:

$\mathrm{X} 0 \mathrm{~B}=+0.33000000$

$\mathrm{YOB}=+0.00000000$

RPUPILB $=+0.50000000$

$\mathrm{CA}[0][0]=+1.0000000000 \mathrm{e}+000$

$\mathrm{cA}[1][1]=+2.0000000000 \mathrm{e}-001$

$C A[1][-1]=+3.0000000000 \mathrm{e}-001$

$\mathrm{cA}[1][0]=+5.0000000000 \mathrm{e}-001$

$\mathrm{CA}[2][2]=-1.0000000000 \mathrm{e}+000$

$\mathrm{CA}[2][0]=+7.5000000000 \mathrm{e}-001$

We have written $\mathrm{cA}[\mathrm{n}][\mathrm{m}]$ for $c_{n m}^{A}$ and $\mathrm{cB}[\mathrm{n}][\mathrm{m}]$ for $c_{n m}^{B}$.

The origin $(\mathrm{XOB}, \mathrm{YOB})$ and pupil radius RPUPILB of circle $\mathrm{B}$ are expressed as fractions of the pupil radius of circle $A$. Thus circle $B$ really has center at $(0.33$ RPUPILA, 0 ) and pupil radius of 0.5 RPUPILA. The origin of circle $\mathbf{A}$ is always at $(0,0)$. Using our unpublished Zernike transformation formulas for translating and scaling to a different origin and different pupil radius, we find that the circle $\mathbf{B}$ Zernike coefficients are:

$\mathrm{cB}[0][0]=+7.8059144500 \mathrm{e}-001$

$\mathrm{cB}[1][1]-5.6656700000 \mathrm{e}-001$

$\mathrm{CB}[1][-1]=+1.5000000000 \mathrm{e}-001$

$\mathrm{cB}[1][0]=-5.1850000000 \mathrm{e}-002$

$\mathrm{cB}[2][2]=-4.9750000000 \mathrm{e}-003$

$\mathrm{cB}[2][1]=+2.4750000000 \mathrm{e}-001$

$\mathrm{cB}[2][0]=+4.6875000000 \mathrm{e}-002$

Now we wish to find the radial Zernike coefficicients for $n>=2$ given the nonradial circle $\mathbf{A}$ and $\mathbf{B}$ Zernike coefficients for $\mathrm{n}>=2$. Thus we start with:

$\mathrm{X} 0 \mathrm{~B}=+0.33000000$

$\mathrm{YOB}=+0.00000000$

RPUPILB $=+0.50000000$

$c A[2][2]=-1.0000000000 \mathrm{e}+000$ 
$\mathrm{cB}[2][2]=-4.9750000000 \mathrm{e}-003$

$\mathrm{cB}[2][1]=+2.4750000000 \mathrm{e}-001$

Using the least squares method just described, we find that:

$\mathrm{XOB}=+0.33000000$

$\mathrm{YOB}=+0.00000000$

RPUPILB $=+0.50000000$

$\mathrm{CA}[0][0]=+4.6196055000 \mathrm{e}-002$

$C A[1][1]=+1.0593660000 \mathrm{e}+000$

$\mathrm{cA}[1][-1]=+4.7252607440 \mathrm{e}-016$

$\mathrm{cA}[1][0]=+7.0740000000 \mathrm{e}-001$

$\mathrm{cA}[2][2]=-1.0000000000 \mathrm{e}+000$

$\mathrm{cA}[2][-2]=+0.0000000000 \mathrm{e}+000$

$\mathrm{cA}[2][1]=+0.0000000000 \mathrm{e}+000$

$\mathrm{cA}[2][-1]=+0.0000000000 \mathrm{e}+000$

$\mathrm{cA}[2][0]=+7.5000000000 \mathrm{e}-001$

$\mathrm{cB}[0][0]=+0.0000000000 \mathrm{e}+000$

$\mathrm{cB}[1][1]=+0.0000000000 \mathrm{e}+000$

$\mathrm{cB}[1][-1]=+0.0000000000 \mathrm{e}+000$

$\mathrm{CB}[1][0]=+0.0000000000 \mathrm{e}+000$

$\mathrm{cB}[2][2]=-4.9750000000 \mathrm{e}-003$

$\mathrm{cB}[2][-2]=+0.0000000000 \mathrm{e}+000$

$\mathrm{CB}[2][1]=+2.4750000000 \mathrm{e}-001$

$\mathrm{cB}[2][-1]=+0.0000000000 \mathrm{e}+000$

$\mathrm{cB}[2][0]=+4.6875000000 \mathrm{e}-002$

The piston, tilts, and focus for circle $\mathbf{A}$ are found so that assuming no piston, tilts and focus in circle $\mathbf{B}$ gives the best match. We have set up the formalism so that the piston, tilts, and focus cannot be recovered since the mirror may be tilted and moved longitudinally in amounts that are not precisely known between measurement sets $A$ and $B$. However, the radial Zernike coefficients $C A[2][0]$ and $\mathrm{cB}[2][0]$ are recovered exactly.

We also find that:

$\mathrm{cA}[2][0]=-0.4337171 \mathrm{cA}[2][2]+1.734868 \mathrm{cB}[2][2]+1.312783 \mathrm{cB}[2][1]+$ other terms

$\mathrm{cB}[2][0]=-0.0290270 \mathrm{cA}[2][2]+0.116108 \mathrm{cB}[2][2]+0.074447 \mathrm{cB}[2][1]+$ other terms

This gives us the detailed sensitivity to the circle A and B nonradial Zernike coeffcicients. ( I have only bothered listing those terms corresponding to the nonzero radial Zernike coefficients). If one plugs in the values for $c A[2][2]$, $\mathrm{cB}[2][2]$, and $\mathrm{cB}[2][1]$, one does get the correct values for $\mathrm{cA}[2][0]$ and $\mathrm{cB}[2][0]$. 
We also get a vector giving the expected errors in all the coefficients assuming that there is the same random fractional error distribution for each nonradial Zernike coefficient and that the individual fractional error distributions are independent.

VECTOR OF THE EXPECTED ERROR MAGNITUDES PER UNIT RANDOM FRACTIONAL VARIATIONS IN ALL THE NONRADIAL COEFFICIENTS

$\mathrm{CA}[2][0] \quad 0.542$

$\mathrm{cB}[2][0] \quad 0.034$

1 (piston) $\quad 0.076$

XA (tiltX) $\quad 0.912$

$Y A$ (tiltY) $\quad 0.000$

$2^{*}\left(X A^{*} X A+Y A^{*} Y A\right)-1 \quad 0.510$

This says that if the fractional error distributions for $c A[2][2], c B[2][2]$, and $c B[2][1]$ all had an rms of 0.1 , that the expected rms error for $\mathrm{CA}[2][0]$ would be 0.054 . Since $c A[2][0]=0.75$, this rms error is $7.2 \%$ of the true value for $\mathrm{cA}[2][0]$.

This numerical example was presented because it is very simple. We have looked at more complicated cases in which higher order radial Zernikes were solved for and have found the method to be very well-behaved. The Zernike coefficients should always be measured in whole shells because when translating and scaling to a different coordinate system, Zernike shells always couple to the same or lower level shells, never to higher shells. 


\section{APPENDIX B: Translational transformation of the Zernike functions}

Since the Zernike functions are a complete set, a Zernike function in one coordinate system can be expressed in terms of the Zernike functions in any other coordinate system. Here we wish to consider only a change of origin or a translation. Scaling transformations due to changes in the normalizing radius are discussed in appendix $\mathrm{C}$, while rotational transformations are trivial. The Zernike functions have the form $Z_{n m}(\rho, \phi)=f_{n m}\left(\rho^{2}\right) \rho^{m} \cos m \phi$ with $n \geq m$ for $m$ $\geq 0$ and $f_{n|m|} \rho|m| \sin |m| \phi$ with $n \geq|m|$ for $m<0$. It is more convenient to work with $\mathrm{N}=\mathrm{n}-|\mathrm{m}|$ rather than $\mathrm{n}$ since the functions $f_{N}^{(m)}(x)$ are of degree $\mathrm{N}$ in $\mathrm{x}=$ $\rho^{2}$ and are defined for $\mathrm{N} \geq 0$. The Rodrigues formula for these functions also has a simpler form. For each $\mathrm{m}$, the functions $f_{N}^{(m)}(x)$ form a complete set over $\mathrm{x}$. The Zernike functions are defined to be orthogonal on the unit circle with the normalization condition $f_{N}^{(m)}(1)=1$. The factor $f_{N}^{(m)}\left(\rho^{2}\right)$ of the Zernike function $Z_{n m}(\rho, \phi)$ is actually a Jacobi polynomial whose argument has been scaled and shifted. A large literature on the properties of Jacobi polynomials exists. Note that this function does not contain all the radial dependence of the corresponding Zernike function except when $m=0$. Any polynomial in $X$ and $Y$ can be represented as a sum of Zernike functions since they can be shown to form a complete set of functions in the $X Y$ plane.

The reason that the factor $\rho|\mathrm{m}|$ exists and that the remainder of the radial dependence depends only upon $\rho^{2}$ can be seen as follows: only terms in $X^{k} Y \mathrm{l}$ with $k+l \geq m \geq 0$ can give rise to terms in $\cos m \phi$ and $\sin m \phi$. Thus if the azimuthal dependence is either cos $\mathrm{m} \phi$ or $\sin \mathrm{m} \phi$, there must be a $\rho^{\mathrm{m}}$ factor. The polynomial in $\rho$ multiplying $\rho^{m_{\text {cos }}} \mathrm{m} \phi$ or $\rho^{m_{\text {sin }}} \mathrm{m} \phi$ must then depend only upon $\rho^{2}$ for we require invariance under the identity transformation $\rho \rightarrow-\rho$ and $\phi \rightarrow 180^{\circ}+\phi$, which maps a point into itself. 
The generators for the translations in $X$ and $Y$ are $\partial / \partial X$ and $\partial / \partial Y$, respectively, where $X$ and $Y$ are the normalized $x$ and $y$ coordinates. We wish to find expansions for $\partial / \partial X Z_{n m}(\rho, \phi)$ and $\partial / \partial Y Z_{n m}(\rho, \phi)$ in terms of the $Z_{n m}(\rho, \phi)$. Define $x=\rho^{2}$. Orthogonality on the unit circle requires that:

$$
\int_{0}^{1} f_{N}^{(m)}(x) f_{N^{\prime}}^{(m)}(x) x^{m} d x=0 \text { for } N \neq N^{\prime}
$$

These are orthogonal polynomials on the unit interval $[0,1]$ with weighting factor $x^{m}$. This property completely determines these polynomials except for their normalization. It also determines the differential equation they obey and their generating function. Using the theory of orthogonal polynomials, they can be shown to obey the differential equation:

$$
\left\{x(1-x) \frac{d^{2}}{d x^{2}}+[(m+1)-(m+2) x] \frac{d}{d x}+\lambda_{N m}\right\} f_{N}^{(m)}(x)=0
$$

where the eigenvalue $\lambda_{\mathrm{Nm}}=\mathrm{N}_{+}(\mathrm{m}+1) \mathrm{N}$.

The theory of orthogonal polynomials starts with a differential equation of the form:

$$
\left(p(x) \frac{d^{2}}{d x^{2}}+q(x) \frac{d}{d x}+\lambda_{n}\right) f_{n}(x)=0
$$

Here $p(x)=p_{2} x^{2}+p_{1} x+p_{0}$ is a polynomial of no higher than the second degree and $q(x)=q_{1} x+q_{0}$ is a polynomial of no higher than the first degree. It may be proved, except for normalization, that:

$$
f_{n}(x)=\frac{1}{w(x)} \frac{d^{n}}{d x^{n}}\left[w(x) p^{n}(x)\right]
$$

where

$$
w(x)=\frac{1}{p(x)} \exp \left[\int \frac{q(x)}{p(x)} d x\right]
$$


It may also be proved that the eigenvalue $\lambda_{n}$ is determined by $p_{2} n(n-$ 1) $+q_{1} n+\lambda_{n}=0$.

From Sturm-Liouville theory, orthogonality on an interval $[a, b]$ with weighting factor $w(x)$ requires that

$$
\left.e^{\int \frac{q(x)}{p(x)} d x} W\left(f_{n}, f_{m}\right)\right|_{a} ^{b}=0
$$

where $W\left(f_{n}, f_{m}\right)$ is the Wronskian of $f_{n}(x)$, and $f_{m}(x)$. Since $w(x)=x^{m}$ for $f_{N}^{(m)}(x)$ :

$$
\exp \left[\int \frac{q(x)}{p(x)} d x\right]=x^{m} p(x)
$$

If $p(x)$ has zeros at 0 and 1 , then the polynomials will be orthogonal on $[0,1]$ with weighting factor $x^{m}$ for $m \geq 0$. Thus $p(x)$ has the form $x(1-x)$ up to a factor. The polynomial $\mathrm{q}(\mathrm{x})$ may be determined so as to make $\mathrm{x}^{\mathrm{m}}$ the weighting factor

The functions $f_{N}^{(m)}(x)$ are given by the Rodrigues formula:

$$
f_{N}^{(m)}(x)=\frac{1}{N ! x^{m}} \frac{d^{N}}{d x^{N}}\left[x^{N+m}(x-1)^{N}\right]
$$

They obey the orthogonality relation:

$$
\int_{0}^{1} f_{N}^{(m)}(x) f_{N^{\prime}}^{(m)}(x) x^{m} d x=\frac{1}{2 N+|m|+1} \delta_{N, N^{\prime}}
$$

Now we are ready to find the expansion for the gradient of a Zernike function in terms of the Zernike functions. Since $X=\rho \cos \phi$ and $Y=\rho \sin \phi$, we have: 


$$
\begin{gathered}
\frac{\partial}{\partial X}=\cos \phi \frac{\partial}{\partial \rho}-\frac{\sin \phi}{\rho} \frac{\partial}{\partial \phi} \\
\frac{\partial}{\partial Y}=\sin \phi \frac{\partial}{\partial \rho}+\frac{\cos \phi}{\rho} \frac{\partial}{\partial \phi}
\end{gathered}
$$

Thus:

$$
\begin{aligned}
& \frac{\partial}{\partial X} f\left(\rho^{2}\right) \cos m \phi=f^{\prime}\left(\rho^{2}\right) \rho^{m+1} \cos (m+1) \phi+\left[\rho^{2} f^{\prime}\left(\rho^{2}\right)+m f\left(\rho^{2}\right)\right] \cos (m-1) \phi \\
& \frac{\partial}{\partial X} f\left(\rho^{2}\right) \sin m \phi=f^{\prime}\left(\rho^{2}\right) \rho^{m+1} \sin (m+1) \phi+\left[\rho^{2} f^{\prime}\left(\rho^{2}\right)+m f\left(\rho^{2}\right)\right] \sin (m-1) \phi \\
& \frac{\partial}{\partial Y} f\left(\rho^{2}\right) \cos m \phi=f^{\prime}\left(\rho^{2}\right) \rho^{m+1} \sin (m+1) \phi-\left[\rho^{2} f^{\prime}\left(\rho^{2}\right)+m f\left(\rho^{2}\right)\right] \sin (m-1) \phi \\
& \frac{\partial}{\partial Y} f\left(\rho^{2}\right) \sin m \phi=-f^{\prime}\left(\rho^{2}\right) \rho^{m+1} \cos (m+1) \phi+\left[\rho^{2} f^{\prime}\left(\rho^{2}\right)+m f\left(\rho^{2}\right)\right] \cos (m-1) \phi
\end{aligned}
$$

Here $\mathrm{f}\left(\rho^{2}\right)$ represents $f_{N}^{(m)}\left(\rho^{2}\right)$. We wish to expand $\frac{d}{d x} f_{N}^{(m)}(x)$ in terms of $f_{N^{\prime}}^{(m+1)}(x)$ with varying $N^{\prime}$ and to expand $\frac{d}{d x} f_{N}^{(m)}(x)+m f_{N}^{(m)}(x)$ in terms of $f_{N^{\prime}}^{(m-1)}(x)$ with varying $N^{\prime}$. Note that there is a problem here when $\mathrm{m}=0$ since $f_{N}^{(m)}(x)$ is not defined for negative $\mathrm{m}$. For the $\mathrm{m}=0$ case, only the cos $\mathrm{m} \phi$ functions exist and we only need $\frac{d}{d x} f_{N}^{(0)}(x)$ in terms of $f_{N}^{(1)}(x)$.

We will prove that for $\mathrm{m} \geq 0$ and $\mathrm{N} \geq 0$

$$
\begin{array}{r}
\frac{d}{d x} f_{N}^{(m)}(x)=[2(N-1)+m+2] f_{N-1}^{(m+1)}(x) \\
{[2(N-2)+m+2] f_{N-2}^{(m+1)}(x)} \\
\vdots \\
{[2(N-N)+m+2] f_{0}^{(m+1)}(x)}
\end{array}
$$

We will also prove that for $\mathrm{m}>0$ and $\mathrm{N} \geq 0$ : 


$$
\begin{array}{r}
x \frac{d}{d x} f_{N}^{(m)}(x)+m f_{N}^{(m)}(x)=[2(N-0)+m] f_{N}^{(m-1)}(x) \\
{[2(N-1)+m] f_{N-1}^{(m-1)}(x)} \\
\vdots \\
{[2(N-N)+m] f_{0}^{(m-1)}(x)}
\end{array}
$$

The expressions $\frac{d}{d x} f_{N}^{(m)}(x)$ and $x \frac{d}{d x} f_{N}^{(m)}(x)+m f_{N}^{(m)}(x)$ are polynomials of degree $\mathrm{N}-1$ and $\mathrm{N}$, respectively. The expansion (B.12) is for $\mathrm{m}$ $\geq 0$ while the expansion (B.13) is for $m>0$. The coefficients in these two expansions are given by:

$$
\frac{\int_{0}^{1}\left(\frac{d}{d x} f_{N}^{(m)}(x)\right) f_{N^{\prime}}^{(m+1)}(x) x^{m+1} d x}{\int_{0}^{1} f_{N^{\prime}}^{(m+1)}(x) f_{N^{\prime}}^{(m+1)}(x) x^{m+1} d x}
$$

and

$$
\frac{\int_{0}^{1}\left(x \frac{d}{d x} f_{N}^{(m)}(x)+f_{N}^{(m)}(x)\right) f_{N^{\prime}}^{(m-1)}(x) x^{m-1} d x}{\int_{0}^{1} f_{N^{\prime}}^{(m-1)}(x) f_{N^{\prime}}^{(m-1)}(x) x^{m-1} d x}
$$

The first integral may be easily evaluated by using the Rodrigues formula formula given earlier and integrating repeatedly by parts. The same approach may be used on the second integral after making the substitution

$$
x \frac{d}{d x} f_{N}^{(m)}(x)+f_{N}^{(m)}(x)=\frac{1}{x^{m-1}} \frac{d}{d x}\left[x^{m} f_{N}^{(m)}(x)\right]
$$

The explicit equations for the expansion of the gradient of a Zernike function when $\mathrm{m} \neq 0$ are: 


$$
\begin{aligned}
& \frac{\partial}{\partial X} Z_{n, \pm m}(\rho, \phi)= \\
& +\left\{[2 n-m] Z_{n, \pm(m+1)}+[2(n-1)-m] Z_{n-1, \pm(m+1)}+\cdots+[2(m+1)-m] Z_{m+1, \pm(m+1)}\right\} \\
& +\left\{[2(n-1)-m+2] Z_{n-1, \pm(m-1)}+[2(n-2)-m+2] Z_{n-2, \pm(m-1)}\right\}\left(1-\delta_{ \pm m,-1}\right) \\
& +\cdots+[2(m-1)-m+2] Z_{m-1, \pm(m-1)} \\
& \frac{\partial}{\partial Y} Z_{n, \pm m}(\rho, \phi)= \\
& \pm\left\{[2 n-m] Z_{n, \mp(m+1)}+[2(n-1)-m] Z_{n-1, \mp(m+1)}+\cdots+[2(m+1)-m] Z_{m+1, \mp(m+1)}\right\} \\
& \mp\left\{[2(n-1)-m+2] Z_{n-1, \mp(m-1)}+[2(n-2)-m+2] Z_{n-2, \mp(m-1)}\right. \\
& \left.+\cdots+[2(m-1)-m+2] Z_{m-1, \mp(m-1)}\right\}\left(1-\delta_{ \pm m,+1}\right)
\end{aligned}
$$

The factors of $\left(1-\delta_{ \pm m,-1}\right)$ and $\left(1-\delta_{ \pm m,+1}\right)$ are present since there is no Zernike function in $\sin m \phi$ with $m=0$. For the case $m=0$ :

$$
\begin{aligned}
& \frac{\partial}{\partial X} Z_{n, 0}(\rho, \phi)=4 n Z_{n,+1}(\rho, \phi)+4(n-1) Z_{n-1,+1}(\rho, \phi)+\cdots+4 Z_{1,+1}(\rho, \phi) \\
& \frac{\partial}{\partial Y} Z_{n, 0}(\rho, \phi)=4 n Z_{n,-1}(\rho, \phi)+4(n-1) Z_{n-1,-1}(\rho, \phi)+\cdots+4 Z_{1,-1}(\rho, \phi)
\end{aligned}
$$

Let $X_{C}$ and $Y_{C}$ be the location of the origin of the coordinate system for the Zernike expansion measured in units of the normalizing radius. Note that $\partial / \partial X_{c}=-\partial / \partial X$ and $\partial / \partial Y_{c}=-\partial / \partial Y$. The operators $\partial / \partial X_{c}$ and $\partial / \partial Y_{c}$ are the generators for infinitesimal translations of the origin of the coordinate system in the $X$ and $Y$ directions, respectively. The operator representing the finite translation $\left(\Delta \mathrm{X}_{\mathrm{c}}, \Delta \mathrm{Y}\right)_{\mathrm{C}}$ is $\exp \left(\Delta \mathrm{X}_{\mathrm{C}} \partial / \partial \mathrm{X}_{\mathrm{C}}+\Delta \mathrm{Y}_{\mathrm{C}} \partial / \partial \mathrm{Y}_{\mathrm{c}}\right)$. Let the matrices $\mathrm{A}$ and $\mathrm{B}$ be given by: 


$$
\begin{aligned}
& \frac{\partial}{\partial X_{c}} Z_{n m}(\rho, \phi)=A_{n, m ; n^{\prime}, m^{\prime}} Z_{n^{\prime} m^{\prime}}(\rho, \phi) \\
& \frac{\partial}{\partial Y_{c}} Z_{n m}(\rho, \phi)=B_{n, m ; n^{\prime}, m^{\prime}} Z_{n^{\prime} m^{\prime}}(\rho, \phi)
\end{aligned}
$$

The matrices $A$ and $B$ are infinite dimensional. However, note that $A_{n, m ; n, m}$ and $B_{n, m ; n}, m^{\prime}$ are zero if n'>n. If the expansion in Zernike functions is truncated at some shell $n$, then translation will not couple to Zernike functions in higher shells. The matrices $A$ and $B$ will then be finite-dimensional and nilpotent. Nilpotency for a matrix A means that there is some non-negative integer $r$ such that $A^{r}=0$. The operators $\partial / \partial X_{c}$ and $\partial / \partial Y_{c}$ couple a Zernike function $Z_{n m}(\rho, \phi)$ either to lower shells or to higher magnitude $|\mathrm{m}|$. The operators $\partial / \partial X_{c}$ and $\partial / \partial Y_{c}$ have the property that $\left(\partial / \partial X_{c}\right)^{r}\left(\partial / \partial Y_{c}\right)^{S} Z_{n m}(\rho, \phi)=0$ whenever $r+s>2 n-|m|$. This means that $\left(\Delta X_{c} \partial / \partial X_{c}+\Delta Y_{c} \partial / \partial Y_{c}\right)^{t} Z_{n m}(\rho, \phi)=0$ whenever $t>2 n-|m|$. Define the matrix $Q=\Delta X_{c} A+\Delta Y_{C} B$. $Q$ is nilpotent since $Q^{t}=0$ whenever $t>2 n$, where $\mathrm{n}$ is the highest shell kept in the expansion. In evaluating $e^{Q}=1+Q+Q^{2} / 2 !+\ldots+Q^{k} / k !+\ldots$, only the first $2 n+1$ terms have to be kept.

Suppose one has a set of functions $f_{N}\left(P ; X_{C}, Y_{C}\right)$ where $P$ is the point and $X_{C}, Y_{C}$ is the origin for the expansion. Also suppose that matrices $A$ and $B$ are defined so that $\partial / \partial X_{C} f_{N}\left(P ; X_{C}, Y_{C}\right)=A_{N, N^{\prime}} f_{N^{\prime}}\left(P ; X_{C}, Y_{C}\right)$ and that $\partial / \partial Y_{C} f_{N}\left(P ; X_{C}, Y_{C}\right)$ $=B_{N, N}{ }^{\prime} f_{N}\left(P ; X_{C}, Y_{C}\right)$. Note that $\partial P / \partial X_{C}=0$ and $\partial P / \partial Y_{C}=0$. If some function $F(P)$ is expanded in terms of these functions with coefficients $a_{N}$ such that $F(P)=\Sigma a_{N}$ $f_{N}\left(P ; X_{C}, Y_{C}\right)$, then $\partial / \partial X_{C} a_{N}=-a_{N^{\prime}} A_{N^{\prime}, N}$ and $\partial / \partial Y_{C} a_{N}=-a_{N^{\prime}} B_{N^{\prime}, N}$. since $\partial / \partial X_{C}$ $F(P)=0$ and $\partial / \partial Y_{C} F(P)=0$.

It is common to number the Zernike functions. They are ordered by shells $n$ where $n \geq 0$ and $-n \leq m<n$. Within each shell, the Zernikes are ordered as follows: $m=+n, m=-n, m=+(n-1), m=-(n-1), \ldots, m=+1, m=-1$, $m=0$. The numbering is usually 1 -based so that the purely radial Zernikes 
$Z_{00}(\rho, \phi), Z_{10}(\rho, \phi), Z_{20}(\rho, \phi), \ldots, Z_{n 0}(\rho, \phi), \ldots$ have $N=1,4,9, \ldots,(n+1)^{2}, \ldots$ Note that this $N$ differs from the $N$ used as a subscript on the functions $f_{N}^{(m)}\left(\rho^{2}\right)$. Eqn (B.17) can alternatively be written as:

$$
\begin{aligned}
& \frac{\partial}{\partial X_{c}} Z_{N}(\rho, \phi)=A_{N, N^{\prime}} Z_{N^{\prime}}(\rho, \phi) \\
& \frac{\partial}{\partial Y_{c}} Z_{N}(\rho, \phi)=B_{N, N^{\prime}} Z_{N^{\prime}}(\rho, \phi)
\end{aligned}
$$




\section{APPENDIX C: Scaling transformation of the Zernike functions}

The Zernike functions $Z_{n m}(\rho, \phi)$ depend upon the normalized radius $\rho=r / R$ and the azimuthal angle $\phi$. We wish to express the Zernike functions with the normalizing radius Rold in terms of the Zernike functions with a different normalizing radius $R$ new. The operator $R \partial / \partial R$ is the generator for an infinitesimal scale transformation. Note that $R \partial / \partial R Z_{n m}(\rho, \phi)=-\rho \partial / \partial \rho Z_{n m}(\rho, \phi)$. The Zernike functions have the form $Z_{n m}(\rho, \phi)=f_{n m}\left(\rho^{2}\right) \rho^{m}$ cosm $\phi$ with $n \geq m$ for $m \geq 0$ and $f_{n|m|} \rho^{|m|} \sin |m| \phi$ with $n \geq m$ for $m<0$. The operator $-\rho \partial / \partial \rho$ only couples Zernike functions of the same $m$. Restrict attention to $m \geq 0$ since the coefficients of the expansion depend only upon $|\mathrm{m}|$. Now for $\mathrm{m} \geq 0$

$$
-\rho \frac{\partial}{\partial \rho} Z_{n m}(\rho, \phi)=\left(-\rho \frac{\partial}{\partial \rho} f_{n m}\left(\rho^{2}\right)\right) \rho^{m} \cos m \phi-m Z_{n m}(\rho, \phi)
$$

so that we need only to expand $\rho d / d \rho f_{n m}\left(\rho^{2}\right)$ in terms of $f_{n m}\left(\rho^{2}\right)$ of the same $m$ but differing $n$. Let $x=\rho^{2}$ so that $-\rho d / d \rho=-1 / 2 x d / d x$. Also, it is more convenient to work with $\mathrm{N}=\mathrm{n}-\mathrm{m}$ rather than $\mathrm{n}$ since the functions $f_{N}^{(m)}(x)$ obey the Rodriguez formula

$$
f_{N}^{(m)}(x)=\frac{1}{N ! x^{m}} \frac{d^{N}}{d x^{N}}\left[x^{N+m}(x-1)^{N}\right]
$$

The functions $f_{N}^{(m)}(x)$ are normalized so that

$$
\int_{0}^{1} f_{N}^{(m)}(x) f_{N^{\prime}}^{(m)}(x) x^{m} d x=\frac{1}{2 N+m+1}
$$

We wish to find the coefficients $a_{N}$ defined by

$$
x \frac{d}{d x} f_{N}^{(m)}(x)=\sum_{N^{\prime}=0}^{N} a_{N^{r}}(N, m) f_{N^{\prime}}^{(m)}(x)
$$

The set of orthogonal polynomials $f_{N}^{(m)}(x)$ is a complete set for each $\mathrm{m}$. $\mathrm{N}$ varies from zero to infinity and is the degree of the polynomial. Since $f_{N}^{(m)}(1)=1$ for all $\mathrm{m}=0$ and $\mathrm{N}=0$, we can integrate by parts to obtain: 
$a_{N^{\prime}}(N, m)=\left(2 N^{\prime}+m+1\right)\left(1-\int_{0}^{1} \frac{1}{N !} \frac{d^{N}}{d x^{N}}\left[x^{N+m}(x-1)^{N}\right]\left(\frac{1}{x^{m}} \frac{d}{d x}\left[x^{m+1} f_{N^{\prime}}^{(m)}(x)\right]\right)\right) d x$

Since $f_{N^{\prime}}^{(m)}(x)$ is a polynomial of degree $N^{\prime}$, it follows that the factor in parentheses in the integrand is also a polynomial of degree $\mathrm{N}$ which we choose to call $p_{N^{\prime}}(x)$. Repeated integration by parts gives:

$$
a_{N^{\prime}}(N, m)=\left(2 N^{\prime}+m+1\right)\left(1-(-1)^{N} \int_{0}^{1} \frac{1}{N !} x^{N+m}(x-1)^{N} \frac{d^{N}}{d x^{N}} p_{N^{\prime}}(x)\right) d x
$$

For $\mathrm{N}^{\prime}<\mathrm{N}$, the $\mathrm{N}^{\text {th }}$ derivative of $\mathrm{p}_{\mathrm{N}^{\prime}}(\mathrm{x})$ vanishes. Thus $\mathrm{a}_{\mathrm{N}^{\prime}}(\mathrm{N}, \mathrm{m})=2 \mathrm{~N}^{\prime}+\mathrm{m}+1$ for $N^{\prime}<N$ and, of course, $a_{N^{\prime}}(N, m)=0$ for $N^{\prime}>N$. Thus we need to evaluate $a_{N^{\prime}}(N, m)$ only for the remaining case $\mathrm{N}^{\prime}=\mathrm{N}$. Now the leading coefficient in $x^{N}$ for $x \frac{d}{d x} f_{N}^{(m)}(x)$ is $\mathrm{N}$ times the leading coefficient of $\mathrm{x}^{\mathrm{N}}$ for $f_{N}^{(m)}(x)$. Thus

$$
x \frac{d}{d x} f_{N}^{(m)}(x)=N f_{N}^{(m)}(x)+\sum_{N^{\prime}=0}^{N-1}\left(2 N^{\prime}+m+1\right) f_{N^{\prime}}^{(m)}(x)
$$

Since $N=n-m$ and since $x d / d x=1 / 2 \rho d / d \rho$, we have

$$
\rho \frac{d}{d \rho} f_{n m}\left(\rho^{2}\right)=2(n-m) f_{n m}\left(\rho^{2}\right)+2 \sum_{N^{\prime}=0}^{N-1}\left(2 n^{\prime}-m+1\right) f_{n m}\left(\rho^{2}\right)
$$

or

$$
-R \frac{\partial}{\partial R} Z_{n m}(\rho, \phi)=(2 n-m) Z_{n m}(\rho, \phi)+2 \sum_{N^{\prime}=0}^{N-1}\left(2 n^{\prime}-m+1\right) Z_{n^{\prime} m}(\rho, \phi)
$$

We thus have

$$
-R \frac{\partial}{\partial R} Z_{n m}(\rho, \phi)=\sum_{n^{\prime}} T_{n n^{\prime}}(m) Z_{n^{\prime} m}(\rho, \phi)
$$

The matrix $T_{n n}(m)$ is a lower triangular matrix which depends upon $m$. Let some function $F(\rho, \phi)$ be expanded in the Zernike functions, so that $F(\rho, \phi)=\Sigma$ $a_{n m} Z_{n m}(\rho, \phi)$. Both the coefficients $a_{m m}$ and the Zernike functions $Z_{n m}(\rho, \phi)$ are implicitly functions of $R$, but $F(\rho, \phi)$ does not depend upon $R$. This implies

$$
R \frac{\partial}{\partial R} a_{n m}=-\sum_{n^{\prime}} a_{n^{\prime} m} T_{n^{\prime} n}(m)
$$


Since the diagonal elements of the lower triangular matrix $T_{n^{\prime} n}(m)$ are all distinct, it may be diagonalized by some similarity transformation $S$ with the property

$$
\sum_{k, l}\left(S^{-1}\right)_{i k} T_{k l} S_{l j}=T_{i i} \delta_{i j}
$$

Let $\mathrm{a}^{(\mathrm{m})}$ be the vector

$$
a^{(m)}=\left[\begin{array}{c}
a_{m+0, m} \\
a_{m+1, m} \\
a_{m+2, m} \\
\vdots
\end{array}\right]
$$

Then

$$
R \frac{\partial}{\partial R} a^{(m)}=-a^{(m)} S T^{(\text {diag })} S^{-1}
$$

This has the solution

$$
a^{(m)}(R)=a^{(m)}\left(R_{o}\right) e^{\log \left(R / R_{o}\right) S^{-1} T^{(d i a g)} S}
$$

or

$$
a^{(m)}(R)=a^{(m)}\left(R_{o}\right) S^{-1}\left(\frac{R}{R_{o}}\right)^{T^{(\text {diag })}} S
$$




\section{APPENDIX D: Translating and scaling the Zernikes using multinomials}

Translating, rotating, and scaling are all trivial for multinomial functions. A multinomial function has the form:

$$
x^{k} y^{l}
$$

where $\mathrm{k}$ and $\mathrm{I}$ are non-negative integers. If we can find simple transformations from Zernike functions to multinomial functions and from multinomial functions to Zernike functions, then we have another way to translate, scale, and rotate. Rotation is trivial for Zernike functions so only the translating and scaling would be done in the multinomial space. We first obtain the transformation from multinomial functions to Zernike functions. We may write:

$$
x^{k} y^{l}=\sum_{n=0}^{\infty} \sum_{m=-n}^{+n} c_{n, m}^{(k, l)} Z_{n, m}(\rho, \phi)
$$

Since the Zernike functions are orthogonal over the unit circle:

$$
c_{n, m}^{(k, l)}=\frac{\iint x^{k} y^{l} Z_{n, m}(\rho, \phi) d A}{\iint\left[Z_{n, m}(\rho, \phi)\right]^{2} d A}
$$

where both integrals are over the unit circle and

$$
d A=\rho d \rho d \phi
$$

Integrations over $d \rho$ are from 0 to 1 , over $d \phi$ are from 0 to $2 \pi$, and over $d A$ are over the unit circle. The integrals in equations (D.5) and (D.6) are needed:

$$
\begin{aligned}
& N_{n, m} \equiv \iint\left[Z_{n, m}(\rho, \phi)\right]^{2} d A=\frac{\pi}{2}\left(1+\delta_{m, 0}\right) \frac{1}{2 n-|m|+1} \\
& \iint x^{k} y^{l}(x+i y)^{|m|} d A= \\
& \int \rho^{k+l+|m|+1} d \rho \sum_{\substack{L=0 \\
l+L \text { even } \\
l+k+m \text { even }}}^{|m|} 2 i^{L}\left(\begin{array}{c}
|m| \\
L
\end{array}\right) B\left(\frac{k+|m|-L+1}{2}, \frac{l+L+1}{2}\right)
\end{aligned}
$$

where 


$$
B(x, y)=\frac{\Gamma(x) \Gamma(y)}{\Gamma(x+y)}
$$

The radial part of the integral in (D.6) has deliberately been left unevaluated so that the angular part of the integration is made explicit.

The Zernike functions are defined by:

$$
\begin{array}{ll}
Z_{n, m}(\rho, \phi)=f_{N}^{(|m|)}\left(\rho^{2}\right) \rho^{|m|} \cos (|m| \phi) & m \geq 0 \\
Z_{n, m}(\rho, \phi)=f_{N}^{(|m|)}\left(\rho^{2}\right) \rho^{|m|} \sin (|m| \phi) & m<0
\end{array}
$$

where $\mathrm{N}=\mathrm{n}-|\mathrm{m}|$.

The Zernike functions have been defined in terms of orthogonal functions defined by the Rodiguez equation:

$$
f_{N}^{(|m|)}(x)=\frac{1}{N ! x^{|m|}} \frac{d^{N}}{d x^{N}}\left[x^{N+|m|}(x-1)^{N}\right]
$$

which obey the orthogonality relation:

$$
\int_{0}^{1} f_{N}^{(|m|)}(x) f_{N^{\prime}}^{(|m|)}(x) x^{|m|} d x=\frac{\delta_{N, N^{\prime}}}{N+|m|+1}
$$

Using the Rodriguez formula (D.8), it is easily shown that

$$
\int_{0}^{1} x^{p+|m|} f_{N}^{(|m|)}(x) d x=\left(\begin{array}{c}
p \\
N
\end{array}\right) B(p+|m|+1, N+1)
$$

This integral is zero unless $p \geq N$. For $k+l+|m|$ even and $|m| \leq k+l \leq 2 n-|m|$, we get the needed radial integrals:

$$
R_{n ! m \mid}^{(k, l)} \equiv \int \rho^{k+l} f_{N}^{(|m|)}\left(\rho^{2}\right) \rho^{|m|} \rho d \rho=\frac{1}{2}\left(\frac{k+l-|m|}{2}\right) B\left(\frac{k+l-|m|}{2}+1, n-|m|+1\right)
$$

Recognizing that 


$$
\begin{aligned}
& \rho^{|m|} \cos (|m| \phi)=\frac{1}{2}\left[(x+i y)^{|m|}+(x-i y)^{|m|}\right] \\
& \rho^{|m|} \sin (|m| \phi)=\frac{1}{2 i}\left[(x+i y)^{|m|}-(x-i y)^{|m|}\right]
\end{aligned}
$$

is where equation (D.6) and its complex conjugate come in. Combining everything gives:

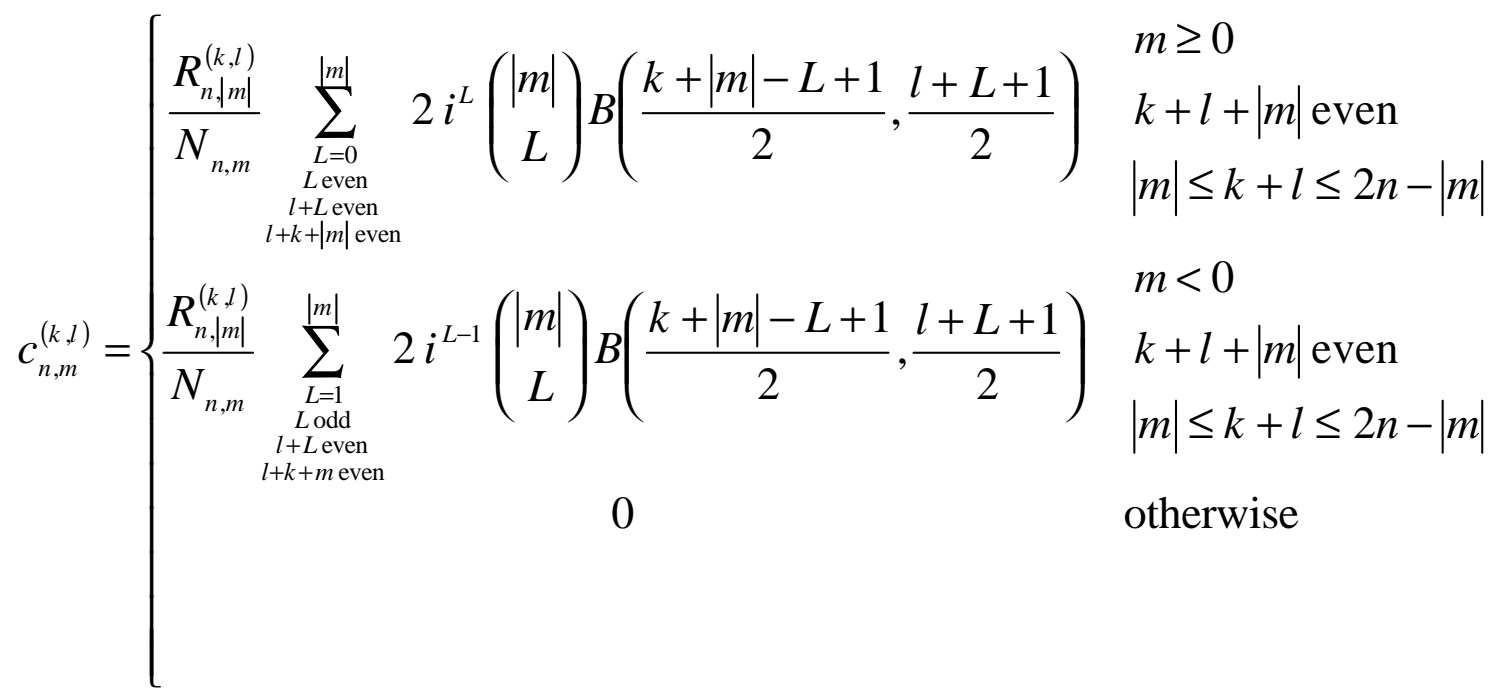

To express a Zernike function in terms of multinomial functions, we start by recognizing that the orthogonal polynomials in Eq. (D.8) are scaled and shifted Jacobi polynomials, which can be defined by their Rodriguez formula as discussed in volume I of The Special Functions and Their Approximations by Luke:

$$
P_{n}^{(\alpha, \beta)}(x)=\frac{(-1)^{n}}{2^{n} n !}(1-x)^{-\alpha}(1+x)^{-\beta} \frac{d^{n}}{d x^{n}}\left[(1-x)^{\alpha+n}(1+x)^{\beta+n}\right]
$$

Luke gives the recursion relation for the Jacobi orthogonal polynomials:

$$
\begin{aligned}
& 2(n+1)(n+\lambda)(2 n+\lambda-1) P_{n+1}^{(\alpha, \beta)}(x)= \\
& (2 n+\lambda)\left[(2 n+\lambda-1)(2 n+\lambda+1) x+\alpha^{2}-\beta^{2}\right] P_{n}^{(\alpha, \beta)}(x) \\
& -2(n+\alpha)(n+\beta)(2 n+\lambda+1) P_{n-1}^{(\alpha, \beta)}(x)
\end{aligned}
$$

where $\lambda=\alpha+\beta+1$. 
Let $\alpha=0, \beta=m, N=n$, and $y=(1+x) / 2$ so that $1-y=(1-x) / 2$. Then $(1-x)(1+x)=$ $-4 y(y-1)$ and $d / d x=1 / 2 d / d y$ We then get the Rodriguez formula:

$$
P_{N}^{(0, m)}(2 y-1)=\frac{1}{N !} y^{-m} \frac{d^{n}}{d y^{n}}\left[(y-1)^{N} y^{m+N}\right]
$$

This is the same Rodriguez formula as Eq. (D.8). Making these substitutions in Eq. (D.14) and replacing $x$ by $2 y-1$ and then renaming $y$ as $x$, we get the recursion relation:

$$
\begin{aligned}
& 2(N+1)(N+m+1)(2 N+m) f_{N+1}^{(m)}(x)= \\
& (2 N+m+1)\left[(2 N+m)(2 N+m+2)(2 x-1)-m^{2}\right] f_{N}^{(m)}(x) \\
& -2 N(N+m)(2 N+m+2) f_{N-1}^{(m)}(x)
\end{aligned}
$$

This recursion relation is easily checked against the first several polynomials calculated from the Rodriguez formula Eq. (D.8):

$$
\begin{array}{cc}
N & f_{N}^{(m)}(x) \\
0 & 1 \\
1 & (m+2) x-(m+1) \\
2 & \frac{1}{2}\left[(m+3)(m+4) x^{2}-2(m+2)(m+3) x+(m+1)(m+2)\right]
\end{array}
$$

Note that the recursion equation (D.16) is consistent with:

$$
f_{N}^{(|m|)}(1)=1
$$


University of California

Lawrence Livermore National Laboratory

Technical Information Department

Livermore, CA 94550

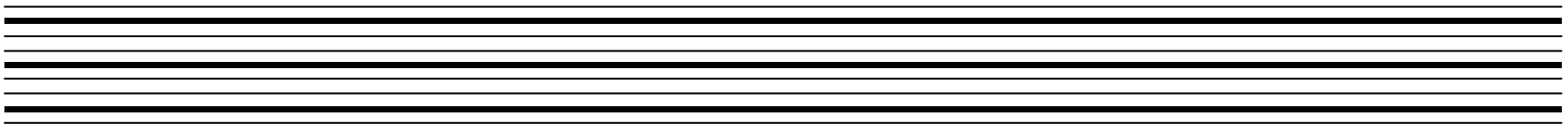

